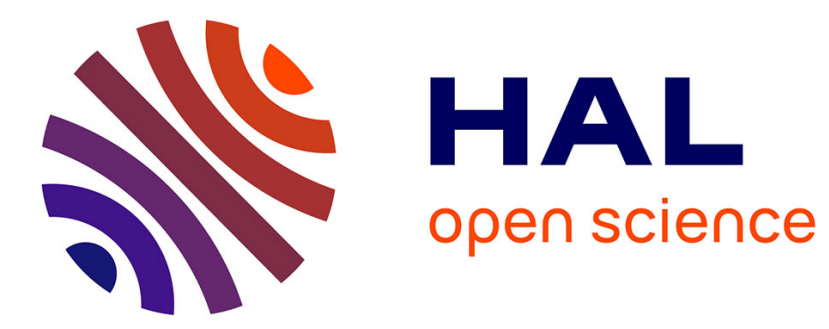

\title{
Une étude des jeux distribués
}

\author{
Julien Bernet, David Janin
}

\section{To cite this version:}

Julien Bernet, David Janin. Une étude des jeux distribués. Revue des Sciences et Technologies de l'Information - Série TSI: Technique et Science Informatiques, 2013, 32 (9-10), pp.1007-1041. hal$00658601 \mathrm{v} 2$

\section{HAL Id: hal-00658601 \\ https://hal.science/hal-00658601v2}

Submitted on 24 Feb 2013

HAL is a multi-disciplinary open access archive for the deposit and dissemination of scientific research documents, whether they are published or not. The documents may come from teaching and research institutions in France or abroad, or from public or private research centers.
L'archive ouverte pluridisciplinaire HAL, est destinée au dépôt et à la diffusion de documents scientifiques de niveau recherche, publiés ou non, émanant des établissements d'enseignement et de recherche français ou étrangers, des laboratoires publics ou privés. 
LaBRI, CNRS UMR 5800

Laboratoire Bordelais de Recherche en Informatique

Rapport de recherche RR-1456-11 (revised february 2013)

Une étude des jeux distribués

24 février 2013

Julien Bernet, David Janin, LaBRI, IPB, Université de Bordeaux 


\section{Table des matières}

1 Jeux distribués $\quad 7$

1.1 Notations . . . . . . . . . . . . . . . . . . . . 7

1.2 Arènes distribuées $\ldots \ldots \ldots \ldots$

1.3 Jeux et stratégies distribuées . . . . . . . . . . . . . . . 10

1.4 Un exemple de jeu distribué . . . . . . . . . . . . . . . . 13

2 Jeux distribués et automates d'arbres 14

2.1 Arbres et automates . . . . . . . . . . . . . . . . 14

2.2 Jeux à condition de gains externes . . . . . . . . . . . . 17

2.3 Théorème d'externalisation . . . . . . . . . . . . . . 20

2.4 Application aux jeux linéaires . . . . . . . . . . . . . . . 21

3 Synchronisation des jeux distribués 23

3.1 Jeux synchrones et synchronisation de jeux asynchrones . . . . . 23

3.2 Synchronisation des jeux linéaires . . . . . . . . . . . . 30

4 Conclusion 33 


\title{
Une étude des jeux distribués
}

\author{
Julien Bernet et David Janin \\ LaBRI, Université de Bordeaux, \\ 351, Cours de la libération, \\ F-33405 Talence cedex, France
}

24 février 2013

Résumé

Dans cet article, nous étudions quelques propriétés des jeux distribués : un modèle abstrait qui vise à capturer divers problèmes formels de synthèse de programmes distribués.

Pour cela, nous examinons en particulier les interconnexions étroites qui existent entre les automate d'arbres alternants et les jeux distribués. L'utilisation du théorème de simulation de Muller et Schupp et d'une notion de composition séquentielle d'automates d'arbres nous permet d'obtenir des outils conceptuels simples et élegants permettant de résoudre, sous certaines hypothèses de linéarité, les jeux distribués.

Parce que les jeux distribués permettent la spécification de comportements synchrones aussi bien qu'asynchrones, nous pouvons aussi énoncer et prouver une intuition assez commune dans le contexte des systèmes distribués : dans le cas d'une capacité mémoire bornée, tout système distribué asynchrone se réduit à un système synchrone. Formellement, nous démontrons que tout jeu distribué asynchrone peut être transformé en un jeu distribué synchrone de même taille qui lui est équivalent au sens où les deux jeux admettent, essentiellement, les mêmes stratégies distribuées à mémoires finies.

In this paper we survey some properties of discrete distributed games : an abstract model that aims at capturing various formalisms for specifying distributed program synthesis problems.

We describe in particular the tight connection that exist between alternating tree automata theory and distributed games. Using Muller and Schupp's simulation theorem and a notion of sequential composition of tree automata, we provide simple and elegant tools to solve, under adequate linearity hypothesis, distributed games.

Moreover, since distributed games allow the specification of both synchronous and asynchronous behaviors, we also state and prove what used to be a common intuition in distributed system design : when the memory capacity is bounded, asychronous systems are essentially synchronous. More formally, we prove that any asynchronous distributed game can be transformed into an equivalent synchronous games in the sense that they essentially both share the same finite state distributed strategies. 


\section{Introduction}

Les jeux distribués dont il est question dans cette article sont issus du travail de thèse du premier auteur [1] sous la direction du second [8]. Leur définition et leur étude, présentées dans une série de conférences [16, 2, 3, 9, 4], visent a offrir un modèle orienté sémantique - c'est à dire indépendant de toute architecture - pour l'étude et l'analyse des problèmes de spécification et de conception de systèmes distribués.

\section{Des systèmes distribués}

Nous considérons ici des systèmes constitués de plusieurs composants, largement autonomes, qui communiquent et interagissent en produisant ainsi, ensemble, un certain comportement global. Ces systèmes sont dit ouverts car ils sont soumis, au sein de leur environnement d'execution, à des sollicitations externes qui influencent en permanence leurs comportements.

Le modèle proposé ici vise à comprendre et à identifier un peu mieux les difficultés intrinsèques liées à la spécification et à la conception de ces systèmes distribués. Plus abstrait, ce modèle généralise de nombreux autres modèles formels de synthèse $[24,23,11]$. Cependant, il n'est pas universel. Plusieurs concepts essentiels dans la mise au point de ces systèmes, tels que par exemple la temporisation des exécutions, sont tout simplement absents. Notre étude s'appuie sur certaines hypothèses simplificatrices, plutôt classiques en méthodes formelles, qui sont présentées ci-dessous. Elles permettent en particulier de décrire les conditions d'application du modèle proposé ici.

1. Nous supposons que le temps inhérent aux systèmes modélisés est discret, divergent et implicite. Plus précisément, nous supposons dans ce modèle que le temps s'écoule de façon discrète, chaque instant suivant l'autre après un délai minimum mais inconnu, ce qui suffit à garantir qu'une infinité d'évènements successifs prennent un temps infini.

2. Nous supposons les données manipulées par ces systèmes en nombre et à valeurs finies. En particulier, la mesure de l'écoulement du temps ne peut pas constituer, du moins en toute généralité, une donnée manipulée par le système.

A chaque instant les systèmes considérés sont caractérisés par des états globaux courants - en nombre fini - et l'évolution de ces systèmes peut être modélisée par une succession de transitions "instantanées" faisant passer le système d'un état global à un autre. En théorie de la concurrence, on se placera donc dans le cadre d'une sémantique par "interleaving" bien distincte de la sémantique "true concurrency", plus riche, qui pourrait être envisagée.

3. Notre approche privilégie la sémantique comportementale des données d'un système plutôt que leur syntaxe (ou leur structuration). Par exemple, qu'importe de savoir si les données d'un système sont structurées en 64 registres de 1 bit ou bien en un seul registre de 64 bits. Dans notre approche, un tel système sera, dans tout les cas, modélisé par un état global de mémoire pouvant prendre $2^{64}$ valeurs. 
4. Nous supposons aussi que les systèmes modélisés peuvent être décrits à l'aide d'un nombre fini de processus distribués communiquants. Pour autant, les architectures de communication reliant les sites de chacun de ces processus restent implicites. Dans notre modèle, abstrait, la distribution des données est modélisée par la notion d'information partielle dont dispose les processus sur chaque site. Tout transfert d'information d'un site à l'autre induit une communication partielle instantanée mais qui reste implicite. Le bénéfice qu'on retire d'une telle approche est que notre modèle inclut aussi donc la modélisation de systèmes distribués, tels que les systèmes à agents mobiles, dont le réseau de communication change au court du temps.

5. Le comportement global des systèmes modélisés est par ailleurs vu comme une succession (potentiellement infinie) d'alternances entre (1) une action globale, incontrôlable, de l'environnement d'exécution, résultant de l'état global du système, et (2) un ensemble d'actions locales (au plus une par processus), contrôlables et indépendantes, définissant les réponses locales de chacun des composants actifs à la sollicitation globale de l'environnement. Cette alternance présuppose implicitement que les systèmes modélisés sont globalement équitables : ni l'environnement, ni les processus, ne peuvent prendre définitivement le contrôle du système.

6. Nous supposons enfi que les systèmes modélisés ont un nombre fini d'états possibles. Cette hypothèse nous interdit, a priori, de traiter le cas des systèmes asynchrones généraux à communication par files d'attentes : la modélisation de ces dernières présupposerait en effet une mémoire non bornée.

\section{Des jeux pour la spécification de programmes}

Notre modèle s'appuie sur la notion de jeu discret [7] qui, en informatique, peut être utilisée pour modéliser toutes les interactions possibles entre les programes à concevoir et l'environnement dans lequel ils évolueront. Dans cette approche, les programmes corrects sont modélisés par des stratégies gagnantes. Pour traiter le cas des systèmes distribués, nous sommes donc amenés à définir des jeux à plus de deux joueurs où s'affrontent un environnement et une équipe de processus distribués.

Dans le détail, les coups des Processus sont définis localement : chaque processus dispose de sa propre arène et y joue comme il le ferait dans un jeu à deux joueurs. En particulier, à partir de toute position locale à son arène, l'ensemble des coups qu'un processus peut jouer est indépendant de la position globale du système, c'est à dire du contexte instantané dans lequel il évolue.

$\mathrm{Au}$ contraire, les coups du joueur Environnement sont globaux, mais peuvent être contraints. Ils représentent bien sûr les sollicitations possibles d'un environnement externe au système modélisé, mais permettent en outre de modéliser les règles auxquelles le système est soumis.

Par exemple, si l'on désire modéliser un canal de communication fiable, l'environnement peut être contraint à recopier la valeur produite en entrée du canal

par le processus émetteur, afin de la rendre disponible en sortie du canal par le processus récepteur. 
Ainsi, dans le modèle proposé, spécifier un système distribué revient à décrire, au moyen d'un jeu distribué, l'ensemble des entrées possibles du système, l'ensemble des comportement locaux possibles des processus, l'ensemble des conséquences globales de ces comportments locaux. La synthèse d'un système distribué répondant à une telle spécification revient alors à choisir parmi les comportements locaux possibles des stratégies locales assurant, ensemble, le bon fonctionnement global du système.

\section{Contexte de recherche et travaux connexes}

Notre modèle s'inspire largement des travaux de Reif et al. [20, 21] dans lesquels la notion d'information partielle est utilisée pour définir un modèle de calculabilité avec informations distribuées.

Dans le contexte des systèmes finis et des spécifications comportementales linéaires, le problème de la synthèse de programmes sur architecture distribuées est formalisé par Pnueli et Rosner [22, 23]. Leurs travaux sont étendus aux spécifications arborescentes par Kupferman et Vardi ă[10]. Dans ces approches, la décidabilité des architectures linéaires est chaque fois démontrée, le cas général étant indécidable. Dans le contexte légèrement différent de la théorie du contrôle, on peut aussi citer aussi les travaux de Wonham et al [12] ou encore les travaux de Vincent [25].

C'est dans les travaux de thèse de Madhusudan [13] qu'on peut trouver une synthèse de la plupart des fondements du domaine. Ce dernier obtient en particulier [14] une extension de la classe des architecture décidables, en s'intéressant au cas de spécifications comportementales composées uniquement de spécifications locales à chaque site. Notre approche généralise ces travaux en ce qu'elle permet aussi de résoudre le cas des architectures pipeline mais dans le cas plus général de comportement partiellement asynchrones.

La théorie des automates utilisés ici est, quant à elle, une variation de la théorie des automates alternants de Muller et Schupp [17, 18] qui offre un outillage conceptuel puissant construit autour de la théorie des automates d'arbres.

\section{Structure de l'article}

Nous présentons, dans une première partie, le modèle des jeux distribués et la notion de stratégies distribuées qui en découle.

Quelques propriétés élémentaires de ces jeux sont exposées. La modélisation implicite des communications par la restriction des coups possibles de l'environnement est illustrée à travers le codage, en terme de jeux distribués, du problème de la synthèse de programmes sur une architecture pipeline.

Dans une seconde partie, les liens étroits qui relient cette définition et la théorie des automates d'arbres alternants de Muller et Schupp sont présentés. Ils offrent un outillage simple qui nous permet, sous certaines hypothèses de linéarité des jeux, de fournir un algorithme de résolution des jeux distribués : un algorithme permettant de décider et de construire, si elles existent, les stratégies distribuées finies gagnantes du jeu fourni en entrée. 
Les constructions qui sous tendent les algorithmes de résolution de problèmes de synthèses distribués $[22,23,12,10]$ ou de résolution des jeux distribués [16] pourraient apparaître comme autant de constructions spécifiques. Elles s'unifient dans notre approche au travers de notions somme toute classiques de composition séquentielle d'automates et de simulation d'automates d'arbres alternants par des automates d'arbres non alternants [18].

La décidabilité du problème de la synthèse de programmes sur les architectures pipeline est, en particulier, une conséquence de la linéarité des jeux distribués qui les modélisent.

Le modèle des jeux distribués permet la modélisation de comportement locaux synchrones aussi bien qu'asynchrones. Il permet donc d'explorer formellement quelques propriétés de la spécification de systèmes distribués dans des contextes synchrones ou asynchrones qui, en l'absence de modèle formel, restent du domaine de l'intuition. Ainsi, le modèle proposée nous permet d'énoncer et de prouver l'intuition suivante : dans le cas de capacités mémoires bornées, tout système distribué asynchrone se comporte, pour l'essentiel, comme un système synchrone.

Nous démontrons dans cet article que si l'on se restreint aux stratégies distribuées à mémoire finies, tout jeu distribué asynchrone peut être normalisé, de façon simple, en un jeu distribué synchrone équivalent au jeu initial (au sens où les stratégies distribuées gagnantes qu'ils induisent sont essentiellement les mêmes).

Il faut cependant apporter un bémol à cette construction, dans la mesure où elle ne préserve pas la linéarité des jeux. Une procédure de (re)linéarisation a posteriori est donc proposée pour remédier à cela.

D'autres approches de résolution de problèmes de synthèses distribués, plus récentes, et qui semblent échapper à notre modèle, sont évoquées en conclusion et qui montrent en un sens quelques limites du modèle.

\section{Jeux distribués}

Nous définissons dans cette section la notion de jeu distribué, ainsi que celle de stratégie distribuée qui l'accompagne. Nous illustrons ensuite ces définitions par la construction de jeux distribués à partir d'architectures dites pipelines, qui nous servira de fil rouge tout au long de notre présentation.

\section{$1.1 \quad$ Notations}

Nous utilisons dans cet article des notations standards en théorie des mots. Nous précisons cependant certaines des ces notations afin d'éviter toute ambiguïté. Pour tout alphabet $A$, on note $A^{*}$ (resp. $A^{\omega}$ ) l'ensemble de tout les mots finis (resp. infinis) sur l'alphabet $A$. On utilise les notations $A^{\infty}=A^{*} \cup A^{\omega}$ et $A^{?}=\{\epsilon\}+A$. Etant donné $L \subseteq A^{*}$, on note aussi $L^{+}$(resp. $L^{\omega}$ lorsque $\epsilon \notin L$ ) l'ensemble des mots finis (respectivement infinis) construit en concaténant un nombre fini (resp. un nombre infini) de mot de $L$. 
Pour tout mot fini $w=a_{1} \ldots a_{n}$, on note $|w|=n$ la longueur du mot $w$. Pour tout mot infini $w$, on note $\inf (w)=\left\{a \in A \mid w \in\left(A^{*} . a\right)^{\omega}\right\}$ l'ensemble des lettres de $A$ qui apparaissent infiniment dans le mots $w$. Etant donné deux ensembles $A$ et $X \subseteq A$, pour tout mot $w \in A^{\infty}$, on note aussi $\pi_{X}(w)$ la projection du mot $w$ sur l'alphabet $X$ c'est à dire le mot obtenu à partir de $w$ en supprimant toutes les lettres qui n'appartiennent pas à $X$.

Etant donnés $n$ ensembles $A_{1}, \ldots, A_{n}$, étant donné $A=A_{1} \times \ldots \times A_{n}$, étant donné un ensemble d'indices $I=\left\{i_{1}, \ldots, i_{k}\right\} \subseteq\{1, \ldots, n\}$ avec $i_{1}<i_{2}<\cdots<$ $i_{k-1}<i_{k}$, on note $A[I]$ l'ensemble $A[I]=A_{i_{1}} \times \ldots A_{i_{k}}$. Pour tout $x \in A$ de la forme $x=\left(a_{1}, \ldots, a_{n}\right) \in A$, on note aussi $x[I]$ le $k$-uplet définit par $x[I]=\left(x_{i_{1}}, \ldots, x_{i_{k}}\right) \in A[I]$. Enfin, pour tout ensemble $P \subseteq A$, on note $P[I]$ l'ensemble défini par $P[I]=\{x[I] \in A[I] \mid x \in P\}$.

Dans le cas où l'ensemble d'indices $I$ est l'ensembles des entiers compris entre $i$ et $j$ inclus on pourra simplifier ces notations en $A[i, j], x[i, j]$ et $P[i, j]$ respectivement (allant jusqu'à noter $A[i], x[i]$ et $P[i]$ lorsque $i=j$ ). Ces notations s'étendent naturellement aux mots sur l'alphabet $A$ de la façon suivante : pour tout mot $w=a_{1} . a_{2} \ldots \in A^{\infty}$, pour tout $I \subseteq\{1, \ldots, n\}$, on note $w[I]=a_{1}[I] \cdot a_{2}[I] . a_{3}[I] \ldots$ Elles s'étendent aussi aux relations : pour toute relation $R \subseteq A \times A$, on note $R[I]$ la relation sur $A[I]$ définit par $R[I]=\{(x[I], y[I]) \in$ $A[I] \times A[I] \mid(x, y) \in R\}$.

En particulier, la notation $w[i, j]$ désignera ici le mot obtenu à partir de $w$ en projetant chacune de ses lettres sur l'alphabet $A[i, j]$.

Le sous-mot de $w$ constitué de la séquence des lettres situées entre la ième à la jème lettre de $w$ sera noté $w(i, j)$ où même $w(i)$ lorsque $i=j$.

\subsection{Arènes distribuées}

Une arène distribuée à $n$ processus est une arène de jeu construite à partir du produit de $n$ arènes simples qui définit les coups possibles d'un joueur Environnement jouant seul contre $n$ joueurs Processus numérotés de 1 à $n$.

\section{Définition 1.2.1 (Arènes de jeux)}

Une arène de jeux simple est une structure de la forme $G=\left\langle P, E, T_{P}, T_{E}\right\rangle$ constituée d'un ensemble $P$ de positions du joueur Processus, un ensemble $E$ de positions du joueur Environnement, un ensemble $T_{P} \subseteq P \times E$ de coups possibles pour le joueur Processus et un ensemble $T_{E} \subseteq E \times P$ de coups possibles pour le joueur Environnement.

Etant données $n$ arènes simples de la forme $G_{i}=\left\langle P_{i}, E_{i}, T_{P, i}, T_{E, i}\right\rangle$, pour chaque $i \in[1, n]$, une arène de jeux distribuée construite à partir des arènes de jeux locales $\left\{G_{i}\right\}_{i \in[1, n]}$ est une arène de jeu de la forme $G=\left\langle P, E, T_{P}, T_{E}\right\rangle$ satisfaisant les propriétés suivantes :

1. Positions de l'environnement : $E=\prod_{i \in[1, n]} E_{i}$,

2. Positions des processus : $P=\prod_{i \in[1, n]}\left(E_{i} \cup P_{i}\right)-\prod_{i \in[1, n]} E_{i}$,

3. Coups des processus : $T_{P}$ est l'ensemble de toutes les paires $(p, e) \in(P \times E)$ telles que, pour tout $i \in[1, n]$ : 
- soit $p[i] \in P_{i}$ et $(p[i], e[i]) \in T_{P, i}$ (le processus $i$ est actif dans la position p),

- soit $p[i] \in E_{i}$ et $p[i]=e[i]$ (le processus $i$ est inactif dans la position $p$ ),

4. Coups de l'environnement $: T_{E}$ est un sous-ensemble de l'ensemble de toutes les paires $(e, p) \in(E \times P)$ telles que, pour tout $i \in[1, n]$ :

- soit $p[i] \in P_{i}$ et $(e[i], p[i]) \in T_{E, i}$ (l'environnement active le processus i),

- soit $p[i] \in E_{i}$ et $p[i]=e[i]$ (l'environnement garde le processus $i$ inactif).

Remarque 1.2.2 La définition des arènes distribuées n'est pas forcément simple en première lecture, et appelle quelques commentaires.

On remarque tout d'abord que les coups du joueur Environnement sont définis globalement. Au contraire, les coups de chaque joueur Processus sont définis localement sur chaque arène. Cela nous permettra, via la notion de stratégie locale, de modéliser le fait que chaque processus n'a qu'une connaissance partielle de la position globale le joueur Environnement pouvant même désactiver certain processus sans qu'ils en aient connaissance.

En effet, à chaque instant, le joueur Environnement a connaissance de la position globale du jeu. Au contraire, à partir d'une position globale $p$, le processus $i$ ne connait a priori que sa propre position $p[i]$.

En particulier, un coup global de l'équipe des processus est constitué de toute combinaison de coups locaux des processus actifs. Sans préjuger de la qualité de la position globale du joueur Environnement qui sera ainsi atteinte - une telle position pourra par exemple être perdante pour l'équipe des processus - toute combinaison de choix locaux est possible.

Remarquons cependant que la définition d'une arène distribuée nous invite aussi à restreindre les coups possibles du joueur Environnement. Certaines combinaisons de coups localement définis pour l'environnement peuvent ne pas être autorisées. On comprend alors qu'on peut forcer l'environnement à transmettre de l'information d'une arène à l'autre. Dans ce cas, le transfert d'information est modélisé implicitement, au niveau sémantique, sans aucune référence $a$ priori à une quelconque architecture de communication.

C'est cette dernière caractéristique qui permet de modéliser, en particulier, les problèmes de synthèse distribués à la Pnueli et Rosner [23, 11], ou bien les problèmes de synthèse de contrôleur à la Wonham [12]. Mais nous pourrions tout aussi bien modéliser des réseaux de processus avec des architectures de communications changeantes. Le modèle proposé est générique.

Lorsque toute les combinaisons possibles de coups locaux de l'environnement sont autorisées, on obtient une arène maximale en un certain sens et qui mérite d'être identifiée. Plus précisément :

Définition 1.2.3 (Produit asynchrone libre)

Dans le cas où l'ensemble $T_{E}$ des coups du joueur Environment de l'arène distribuée $G$ est maximal, l'arène est appelée le produit asynchrone libre des arènes $\left\{G_{i}\right\}_{i \in[1, n]}$ et elle est notée $G_{1} \otimes G_{2} \otimes \cdots \otimes G_{n}$. 
Remarque 1.2.4 Toute arène, simple ou distribuée, peut être vue comme un graphe biparti orienté dont les sommets sont les positions de l'environnement et du ou des processus, et dont les arcs sont les coups possibles entres ces positions. On peut parler de plongement d'une arène dans une autre au sens des plongements de graphes, qui préservent en outre la partition des sommets.

Constatons que toute arène distribuée $G$ définie comme ci-dessus peut être plongée dans le produit asynchrone libre $G_{1} \otimes G_{2} \otimes \cdots \otimes G_{n}$.

Plus généralement, les arènes distribuées étant construites à partir d'un produit d'arènes locales, on peut définir une opération inverse de projection d'une arène distribuée. L'arène distribuée initiale se plongeant alors dans le produit de ses projections.

Formellement :

Définition 1.2.5 (Projection)

Pour tout sous-ensemble d'indices $I \subseteq[1, \cdots, n]$, la projection $G[I]$ de $G$ sur ses composantes d'indice $I$ est définie comme l'arène $G[I]=\left\langle P^{\prime}, E^{\prime}, T_{P}^{\prime}, T_{E}^{\prime}\right\rangle$ avec $P^{\prime}=P[I]-E[I]$ (éventuellement strictement incluse dans $\left.P[I] !\right), E^{\prime}=E[I]$, $T_{P}^{\prime}=T_{P}[I] \cap\left(P^{\prime} \times E^{\prime}\right)$ et $T_{E}^{\prime}=T_{E}[I] \cap\left(E^{\prime} \times P^{\prime}\right)$.

Remarque 1.2.6 Avec cette définition, on constate que l'arène distribuée $G$, initialement définie à partir des arènes $\left\{G_{i}\right\}_{i \in[1, n]}$, peut maintenant être définie à partir des arènes $\{G[i]\}_{i \in[1, n]}$; pour tout $i \in[1, n]$ l'arène locale $G[i]$ peut être plongée dans l'arène locale $G_{i}$ et l'arène $G$ se plonge dans $G[1] \otimes G[2] \cdots \otimes G[n]$.

Plus généralement, pour tout sous-ensemble non vide $I \subset\{1, \ldots, n\}$, étant donné $\bar{I}=\{1, \ldots, n\}-I$, l'arène $G$ distribuée sur $n$ processus peut, tout aussi bien, être vue comme une arène distribuée de deux processus produite à partir des arènes locales $G[I]$ et $G[\bar{I}]$. Ainsi, l'arène $G$ se plonge dans $G[I] \otimes G[\bar{I}]$ et chacune des arènes $G[I]$ et $G[\bar{I}]$ peut, le cas échéant, être vue comme une arène distribuée plus finement.

Autrement dit, la définition d'une arène distribuée à partir de composants locaux est, en un certain sens, associative. C'est cette propriété qui nous permet par la suite de réduire la plupart des preuves portant sur des jeux distribués à $n$ processus à des preuves portant sur les jeux distribués à deux processus.

\subsection{Jeux et stratégies distribuées}

Pour définir la notion de jeux distribué, rappelons tout d'abord la définition d'un jeu simple. La distinction entre jeux distribués et jeux simples sera ensuite obtenue en restreignant les stratégies admissibles de l'équipe des processus à une certaine famille de stratégies : les stratégies distribuées.

Définition 1.3.1 (Jeu et partie)

Un jeu $G=\left\langle P, E, T_{E}, T_{P}, e_{0}, \mathcal{W}\right\rangle$ est défini comme étant une arène de jeu $\left\langle P, E, T_{E}, T_{P}\right\rangle$ équipée d'un état initial $e_{0} \in E$ et d'un ensemble de mots $\mathcal{W} \subseteq$ $(E+P)^{\omega}$ définissant les conditions de victoires pour le (les) joueur(s) Processus. 
Une partie, à partir de la position initiale $e_{0} \in E$, est définie comme un chemin (au sens de la théorie des graphes orientés) dans l'arène $G$ issu de la position $e_{0}$.

Les parties d'un jeu sont construites de la façon suivante. A tout instant, dans une position courante $x \in P \cup E$, soit $x \in E$ et c'est au tour de l'environnement de jouer en choisissant une position $y \in P$ telle que $(x, y) \in T_{E}$, ou bien $x \in P$ et c'est alors à l'équipe des processus de jouer en choisissant une position $y \in E$ telle que $(x, y) \in T_{P}$.

L'avancement du jeu définit donc bien un mot $w \in E .(P . E)^{*} . P^{\text {? }}$, issue de la position initiale $e$, qui peut être prolongé, selon le cas, par l'environnement ou le (ou l'équipe des) processus. La partie qui sera effectivement jouée à partir de la position initiale dépend donc naturellement des stratégies suivies par chacun des joueurs.

\section{Définition 1.3.2 (Stratégie)}

Une stratégie (déterministe) pour l'environnement est une fonction partielle $\tau: E .(P . E)^{*} \rightarrow P$ telle que, pour tout mot w.e $\in \operatorname{dom}(\tau)$ avec $w \in(E . P)^{*}$ et $e \in E$, on $a(e, \tau(w . e)) \in T_{E}$.

De la même façon, une stratégie (déterministe) pour le (ou l'équipe des) processus est une fonction partielle $\sigma:(E . P)^{+} \rightarrow E$ telle que, pour tout mot $w . p \in \operatorname{dom}(\sigma)$ avec $w \in E .(P . E)^{*}$ et $p \in P$, on a $(p, \sigma(w . p)) \in T_{P}$.

Une partie $w$ est dite compatible avec la stratégie $\sigma$ (resp. la stratégie $\tau$ ) lorsque, en notant $w(i)$ la ième lettre du mot $i$ et $w(1, i)$ le prefix de $w$ de longueur $i$, pour tout entier $n$ tel que $0<n<|w|$, si $w(i) \in P$ alors $w(i+1)=$ $\sigma(w(1, i))$ (resp. si $w(i) \in E$ alors $w(i+1)=\tau(w(1, i))$ ).

On dit alors que la stratégie $\sigma$ des processus (resp. la stratégie $\tau$ de l'environnement) est une stratégie gagnante dans le jeu $G$ à partir de la position initiale $e_{0}$ et avec la condition de gain $\mathcal{W}$ lorsque toute partie maximale issue de $e_{0}$ et compatible avec la stratégie $\sigma$ (resp. la stratégie $\tau$ ) est soit finie et termine sur une position de l'environnement (resp. des processus), soit infinie et appartient $\grave{a} \mathcal{W}$ (resp. n'appartient pas à $\mathcal{W}$ ).

Cette définition plutôt générale ne permet pas de conclure quant à l'existence de stratégie gagnante pour l'environnement ou les processus. Le cas particulier des jeux finis est plus intéressant de ce point de vue. Le jeu $G$ est dit fini lorsque $P$ et $E$ sont deux ensembles finis et lorsque $\mathcal{W}$ est un langage $\omega$-régulier, i.e. définissable par un automate d'états fini de mots infinis.

\section{Définition 1.3.3 (Stratégie finie)}

Etant donné un jeu fini $G=\left\langle P, E, T_{P}, T_{E}, e_{0}, \mathcal{W}\right\rangle$, Une stratégie des processus $\sigma$ est dite stratégie finie ou stratégie à mémoire finie lorsque qu'il existe un quadruplet $\mathcal{M}=\left\langle M, m_{0}, \mu: M \times(P \cup E) \rightarrow M, h: M \times P \rightarrow E\right\rangle$, appelé la mémoire de $\sigma$, avec $M$ un ensemble fini d'états, $m_{0} \in M$ un état initial, $\mu$ une fonction de transition et $h$ une fonction de choix telle que, pour toute partie $w \cdot p \in \operatorname{dom}(\sigma)$ on a $\sigma(w \cdot p)=h\left(\mu^{*}\left(m_{0}, w\right), p\right)$ avec $\mu^{*}$ inductivement défini par 
$\mu^{*}(m, \epsilon)=m$ et $\mu^{*}(m, w \cdot x)=\mu\left(\mu^{*}(m, w), x\right)$ pour tout état $m \in M$, tout mot $w \in(E \cup P)^{*}$ et toute position $x \in(E \cup P)$.

Avec ces définitions :

\section{Théorème 1.3.4 ([15])}

Dans tout jeu fini à deux joueur l'un des deux joueurs admet une stratégie gagnante à mémoire finie.

Autrement, dit, non seulement les jeux finis a deux joueurs sont déterminés, i.e. au moins un joueur admet une stratégie gagnante, mais ceci reste vraie même en restreignant l'ensemble des stratégies admissibles aux stratégies à mémoires finies.

Une arène de jeux distribués apparaît donc comme un cas particulier d'arène de jeux discrets à deux joueurs qui jouent chacun leur tour. Les définitions standards de parties et de stratégies s'étendent sans difficultés. Les composants d'une arène distribuée n'étant, ni plus ni moins, que des arènes de jeux classiques, on dispose donc aussi de notions de parties et de stratégies.

Sur une arêne distribuée, on distingue les parties ou stratégies dites globales, qui se jouent dans le jeu distribué dans son ensemble, des parties ou des stratégies dites locales, qui se jouent dans les projections des jeux distribués. La notion de stratégie distribuée (pour l'équipe des processus) est alors définie comme un ensemble de stratégies locales, une par processus, qui définissent, ensemble mais sans communication, une stratégie globale.

Plus précisément, la vue locale du processus $i \in[1, n]$ d'une partie globale dans un jeu distribué $G=\left\langle P, E, T_{P}, T_{E}, e_{0}, \mathcal{W}\right\rangle$ à $n$ processus est définie par une fonction

$$
\text { view }_{i}:(E \cdot P)^{*} \cdot E^{?} \rightarrow\left(E_{i} \cdot P_{i}\right)^{*} \cdot E_{i}^{?}
$$

inductivement définie par view $(\epsilon)=\epsilon$, pour toute position $x \in P \cup E$, view $(x)=$ $x[i]$, et pour toute partie de la forme $w . x . y$ avec $x$ et $y \in P \cup E$, view $(w \cdot x \cdot y)=$ view $_{i}(w \cdot x)$ lorsque $x[i]=y[i]$ ou bien $\operatorname{view}_{i}(w \cdot x \cdot y)=\operatorname{view}_{i}(w \cdot x) \cdot y[i]$ autrement.

\section{Définition 1.3.5}

Une stratégie globale $\sigma$ pour l'équipe des processus sur un jeu distribué $G$ à $n$ processus est dite stratégie distribuée lorsque, pour chaque indice $i \in[1, n] i l$ existe une stratégie $\sigma_{i}:(E[i] . P[i])^{+} \rightarrow E[i]$ pour le processus $i$ dans le jeu $G[i]$, appelée stratégie locale pour le processus $i$, de telle façon que, pour toute partie globale de la forme $w \cdot p \in(E \cdot P)^{+}$, étant donné l'ensemble $I \subseteq\{1, \ldots, n\}$ des processus actifs dans la position $p, \sigma(w \cdot p)=e$ si et seulement si $e[i]=$ $\sigma_{i}\left(\right.$ view $\left._{i}(w) \cdot p[i]\right)$ lorsque $i \in I$ et $e[i]=p[i]$ dans le cas contraire.

On convient dans ce cas de noter $\sigma_{1} \otimes \sigma_{2} \otimes \cdots \otimes \sigma_{n}$ une telle stratégie distribuée.

Résoudre un jeu distribué, c'est alors vérifier que soit l'environnement admet une stratégie globale gagnante, soit l'équipe des processus admet une stratégie distribuée gagnante. 
Remarque 1.3.6 Bien entendu, toute stratégie distribuée est une stratégie globale. Par contre, la réciproque est fausse. Il existe des stratégies globales qui ne peuvent être distribuées. Il résulte de ce fait que les jeux distribués ne sont pas déterminés. On peut facilement construire un jeu distribué à deux processus qui admet au moins une stratégie gagnante pour l'équipe des processus, mais dont aucune de ces stratégies n'est distribuable.

\subsection{Un exemple de jeu distribué}

Une architecture distribuée telle que définie par Pnueli et Rosner [23] est un ensemble de sites $I$ connectés ensemble par un ensemble $C$ de canaux (unidirectionnels) de communication. Ces canaux relient les sites entre eux (cas des communications internes), ou bien constituent des entrées de l'environnement vers un site ou des sorties d'un site vers l'environnement. La bande passante de chaque canal $c \in C$ est caractérisée par un alphabet fini $X_{c}$ qui donne l'ensemble des valeurs possibles que le canal peut transmettre en une étape élémentaire de communication.

Un exemple typique d'architecture distribuée est l'architecture pipeline dont les sites sont totalement ordonnés, chaque site prenant ses entrées de la sortie du site qui le précède ou de l'environnement s'il est le premier, le dernier site produisant dans l'environnement une sortie.

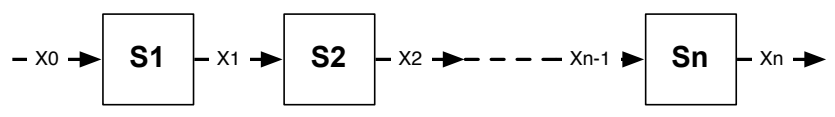

FIGURE 1 - L'architecture pipeline

Dans cette architecture, à chaque instant, chaque site $i \in[1, n]$ reçoit une valeur $x_{i-1} \in X_{i-1}$ et produit une valeur $x_{i} \in X_{i}$. Un programme pour chaque site $i$ est donc une fonction séquentielle $f_{i}: X_{i-1}^{*} \rightarrow X_{i}^{*}$ telle que pour toute entrée $u \in X_{i-1}^{*}$ on a $\left|f_{i}(u)\right|=|u|$.

Le comportement d'une architecture ainsi programmée consiste alors, pour chaque séquence d'entrées $w \in X_{0}^{*}$, à produire la sortie $v \in X_{n}^{*}$ définie par

$$
v=f_{n} \circ f_{n-1} \circ \cdots \circ f_{1}(w)
$$

Montrons maintenant comment, pour chaque architecture pipeline $\mathbb{A}$ à $n$ sites nous pouvons fabriquer une arène distribuée $G_{\mathbb{A}}=\left\langle P, E, T_{P}, T_{E}\right\rangle$ a $n$ processus de telle sorte que les stratégies locales des processus codent les programmes de chaque site, les parties globales joué sur le jeu distribué codant alors le comportement globale de l'architecture pipeline.

Pour faire cela, il suffit de prendre $P=X_{0} \times \ldots \times X_{n-1} ; E=X_{1} \times \ldots \times X_{n}$, les coups locaux de chaque processus $i$ étant définit par $T_{P, i}=X_{i-1} \times X_{i}$ et les coups globaux de l'environnement étant définis par $\left(\left(v_{1}, \ldots, v_{n}\right),\left(v_{0}^{\prime}, \ldots, v_{n-1}^{\prime}\right)\right) \in T_{E}$ lorsque $v_{i}^{\prime}=v_{i}$ pour tout $i \in\{1, \ldots, n-1\}$ et $v_{0}^{\prime} \in X_{0}$.

On vérifie que dans cette arène, à chaque coup, chaque processus lit la valeur d'entrée qui lui est proposée et produit (sans contrainte) la sortie qu'il souhaite. 
Autrement dit, les stratégies locales des processus sont bien des codage des fonctions séquentielles.

Remarquons aussi que les coups de l'environnement sont restreints de telle sorte que, s'il peut effectivement choisir à chaque coup une valeur d'entrée au système (à choisir dans $X_{0}$ ), il est par ailleurs forcé de transmettre chaque valeur produite par le processus $i$ avec $i<n$ au processus qui le suit, numéroté $i+1$.

Les stratégies locales des joueurs Processus sont exactement des fonctions séquentielles $f_{i}: X_{i-1}^{*} \rightarrow X_{i}$. Les stratégies combinées des processus codent donc le comportement globale $f: X_{0}^{+} \rightarrow X_{1} \times \cdots \times X_{n}$ de l'architecture.

Spécifier un problème de synthèse distribuée $[23,11]$ c'est donc spécifier (par exemple à l'aide d'un automate d'arbres infinis) le comportement global attendu. Dans notre codage, cela revient à spécifier un ensemble régulier de stratégies gagnantes pour l'équipe des processus. Cela conduit donc à définir un jeu distribué fini avec condition de gains externe (voir Section 2.2 pour plus de détail) dont on montrera qu'il est équivalent à un jeu distribué fini avec condition de gain interne (voir Théorème 2.2.3). Le jeu produit étant par ailleurs linéaire (voir Section 3.2) on montre enfin qu'il peut effectivement être résolu.

\section{Jeux distribués et automates d'arbres}

Nous présentons ici les connexions étroites qui existent entre la théorie maintenant classique des automates d'arbres et la résolution des jeux distribués dans le cas des architectures dites linéaires. Cette partie reprend pour l'essentiel la communication des deux auteurs présentée en 2005 [2]. Les automates d'arbres manipulés ici sont une variante de la définition des automates arbres alternants de Muller et Schupp [17] qui peuvent simplement se combiner séquentiellement.

\subsection{Arbres et automates}

On présente ici une variation de la notion d'arbre et de la notion d'automate d'arbres associée qui se prête particulièrement bien à l'analyse et au traitement des jeux distribués.

\section{Définition 2.1.1 (Arbre)}

Etant donné deux alphabets finis $D$ et $\Sigma$, un $(D, \Sigma)$-arbre est une fonction partielle $D^{*} \rightarrow \Sigma$ dont le domaine, clos par préfixe, contient au moins le mot vide $\epsilon$. Dans la suite, les éléments de $\Sigma$ sont appelés étiquettes et les éléments de $D$ sont appelés directions.

La définition d'automate suivante, variation sur la définition originale de Muller et Schupp des automates alternants [17], permet d'obtenir une notion d'automates alternants qui se comportent comme des transducteurs d'arbres. Les calculs d'un automate alternant sur un arbre étant eux-même des arbres, ils pourront être composés séquentiellement. 
Définition 2.1.2 (Automate alternant et automate non déterministe) Un automate alternant de $(D, \Sigma)$-arbres est un tuple de la forme :

$$
\mathcal{A}=\left\langle Q=Q^{\forall} \uplus Q^{\exists}, D, \Sigma, q_{0}, \delta=\delta^{\forall} \cup \delta^{\exists}, A c c \subseteq Q^{\omega}\right\rangle
$$

où $Q$ est un ensemble fini d'états partitionné en deux sous-ensembles $Q^{\forall}$ et $Q^{\exists}$ d'états respectivement appelés états universels et états existentiels, $q_{0} \in Q^{\exists}$ est l'état initial, $\delta^{\forall}: Q^{\forall} \times D \rightarrow \mathcal{P}\left(Q^{\exists}\right)$ et $\delta^{\exists}: Q^{\exists} \times \Sigma \rightarrow \mathcal{P}\left(Q^{\forall}\right)$ sont les fonctions de transition, et Acc $\subseteq Q^{\exists} \cdot\left(Q^{\forall} \cdot Q^{\exists}\right)^{\omega}$, critère d'acceptance, est un langage $\omega$ régulier [19].

Un automate d'arbres $\mathcal{A}$ est dit automate non déterministe (ou plus explicitement automate non alternant) lorsque, pour tout état universel $q \in Q^{\forall}$, toute direction $d \in D$, on $a\left|\delta^{\forall}(q, d)\right| \leq 1$.

Enfin, la taille d'un automate d'arbres $\mathcal{A}$, notée $|\mathcal{A}|$, est définie ${ }^{1}$ comme étant la somme des cardinaux de ses ensembles d'états.

Cette définition, qui n'est qu'une variation sur la définition des automates alternants de Muller et Schupp [17], permet de définir les calculs d'automates alternants sur les arbres comme étant eux-mêmes des arbres. Plus précisément, distinguer entre état universel et état existentiel revient à mettre les spécifications de transitions alternantes dans la définition de Muller et Schupp sous forme normale disjonctive.

Cas particulier, lorsque $D=\{1,2\}$ pour coder les arbres binaires, un automate d'arbres binaires non déterministe $\mathcal{A}=\left\langle Q, \Sigma, q_{0}, \delta, A c c\right\rangle$ plus classique avec $\delta: Q \times \Sigma \rightarrow \mathcal{P}(Q \times Q)$ pourra être codé par l'automate non alternant $\mathcal{B}=\left\langle Q^{\forall}, Q^{\exists}, D, \Sigma, q_{0}^{\prime}, \delta^{\forall}, \delta^{\exists}, A c c^{\prime}\right\rangle$ défini par $Q^{\exists}=Q$ et $Q^{\forall}=Q \times Q$ pour les états, $\delta^{\exists}(q, a)=\left\{\left(q_{1}, q_{2}\right) \in Q^{\forall}:\left(q_{1}, q_{2}\right) \in \delta(q, a)\right\}, \delta^{\forall}\left(\left(q_{1}, q_{2}\right), 1\right)=\left\{q_{1}\right\}$ et $\delta^{\forall}\left(\left(q_{1}, q_{2}\right), 2\right)=\left\{q_{2}\right\}$ pour les fonctions de transition, et $A c c^{\prime}=\{w \in$ $\left.\left(Q^{\forall} \cup Q^{\exists}\right)^{\omega}: \pi_{Q}(w) \in A c c\right\}$ pour le critère d'acceptance.

\section{Définition 2.1.3 (Calcul)}

Un calcul de l'automate $\mathcal{A}=\langle Q, D, \Sigma, i, \delta$, Acc $\rangle$ sur un $(D, \Sigma)$-arbret $: D^{*} \rightarrow \Sigma$ est un arbre

$$
\rho:\left(D \times Q^{\exists}\right)^{*} \rightarrow Q^{\forall}
$$

d'étiquettes $Q^{\forall}$ et de directions $D \times Q^{\exists}$, tel que $\rho(\epsilon) \in \delta^{\exists}\left(q_{0}, t(\epsilon)\right)$ et, pour tout $w \in \operatorname{dom}(\rho)$, pour toute direction $d \in D$ telle que $w[D] . d \in \operatorname{dom}(t)$, pour tout état (existentiel) $q \in Q^{\forall}$ tel que $q \in \delta^{\forall}(\rho(w), d)$, on a $\rho(w .(d, q)) \in$ $\delta^{\exists}(q, t(w[D] . d))$.

La séquence states $(\rho, w) \in\left(Q^{\forall} \cdot Q^{\exists}\right)^{\omega}$ des états de l'automate $\mathcal{A}$ rencontrés le long d'une branche infinie $w$ du calcul $\rho:\left(D \times Q^{\exists}\right)^{*} \rightarrow Q^{\forall}$ est inductivement définie par states $(\rho, \epsilon)=\rho(\epsilon)$ et, pour tout $w \in \operatorname{dom}(\rho)$ et toute paire $(d, q) \in\left(D \times Q^{\exists}\right)$ telle que $w \cdot(d, q) \in \operatorname{dom}(\rho)$, par states $(\rho, w \cdot(d, q))=$ $\operatorname{states}(\rho, w) \cdot q \cdot \rho(w \cdot(d, q))$.

1. de façon plutôt sommaire; notre objet n'est pas de faire une étude précise de la complexité des outils et concepts proposés 


\section{Définition 2.1.4 (Calcul acceptant et langage)}

Un calcul $\rho:\left(D \times Q^{\exists}\right)^{*} \rightarrow Q^{\forall}$ de l'automate $\mathcal{A}$ sur un arbre $t: D^{*} \rightarrow \Sigma$ est appelé calcul acceptant lorsque pour toute branche infinie $w \in\left(D \times Q^{\exists}\right)^{\omega}$ de $\rho$ on a states $(\rho, w) \in$ Acc.

Un arbre $t$ est accepté par l'automate $\mathcal{A}$ lorsqu'il existe un calcul acceptant de l'automate $\mathcal{A}$ sur l'arbre $t$.

$L$ 'ensemble $L(\mathcal{A})$ de tous les arbres acceptés par l'automates $\mathcal{A}$ est le langage reconnu par l'automate $\mathcal{A}$.

Bien que définis de façon inhabituelle, ces automates se traduisent facilement en automates de Muller et Schupp [17, 18] et, réciproquement, les automates de Mullet et Schupp se traduisent facilement en ces automates. De plus, ces inter-traductions préservent le caractère non alternant de ces automates. Ainsi, le théorème de simulation de tout automate alternant par un automate non alternant reste valide.

Théorème 2.1.5 (d'après [17])

Pour tout automate alternant $\mathcal{A}$ il existe un automate non alternant $\mathcal{A}^{\prime}$ tel que $L\left(\mathcal{A}^{\prime}\right)=L(\mathcal{A})$ avec $\left|\mathcal{A}^{\prime}\right| \leq 2^{2^{|\mathcal{A}|}}$.

Les calculs des automates étant des arbres, on peut maintenant composer séquentiellement deux automates.

\section{Définition 2.1.6 (Composition séquentielle d'automates)}

Etant donné deux automates d'arbres $\mathcal{A}_{1}=\left\langle Q_{1}, D_{1}, \Sigma_{1}, q_{0,1}, \delta_{1}, A c c_{1}\right\rangle$ et $\mathcal{A}_{2}=$ $\left\langle Q_{2}, D_{2}, \Sigma_{2}, q_{0,2}, \delta_{2}, A c c_{2}\right\rangle$ tels que l'automate $\mathcal{A}_{2}$ est non alternant avec $D_{2}=$ $D_{1} \times Q_{1}^{\exists}$ et $\Sigma_{2}=Q_{1}^{\forall}$, on définit la composition séquentielle de l'automate $\mathcal{A}_{1}$ suivi de l'automate $\mathcal{A}_{2}$ comme étant l'automate sur les $\left(D_{1}, \Sigma_{1}\right)$-arbres

$$
\mathcal{A}_{2} \circ \mathcal{A}_{1}=\left\langle\widetilde{Q}, D_{1}, \Sigma_{1}, \widetilde{q_{0}}, \widetilde{\delta}, \widetilde{A c c}\right\rangle
$$

avec :

$$
\begin{aligned}
& \text { - } \widetilde{Q^{\exists}}=Q_{1}^{\exists} \times Q_{2}^{\exists} ; \widetilde{Q^{\forall}}=Q_{1}^{\forall} \times Q_{2}^{\forall} ; \\
& \text { - } q_{0}=\left(q_{0,1}, q_{0,2}\right) \text {, } \\
& \text { - }\left(q_{1}^{\prime}, q_{2}^{\prime}\right) \in \widetilde{\delta^{\forall}}\left(\left(q_{1}, q_{2}\right), d\right) \Leftrightarrow\left\{\begin{array}{l}
q_{1}^{\prime} \in \delta^{\forall}\left(q_{1}, d\right) \\
\left\{q_{2}^{\prime}\right\}=\delta_{2}^{\forall}\left(q_{2},\left(d, q_{1}^{\prime}\right)\right)
\end{array}\right. \\
& \text { - }\left(q_{1}^{\prime}, q_{2}^{\prime}\right) \in \widetilde{\delta}^{\exists}\left(\left(q_{1}, q_{2}\right), a\right) \Leftrightarrow\left\{\begin{array}{l}
q_{1}^{\prime} \in \delta^{\exists}\left(q_{1}, a\right) \\
q_{2}^{\prime} \in \delta_{2}^{\exists}\left(q_{2}, q_{1}^{\prime}\right)
\end{array}\right. \\
& \text { - } \widetilde{A c c}=\left\{w \in \widetilde{Q}^{\omega} \mid w[1] \in A c c_{1} \wedge w[2] \in A c c_{2}\right\}
\end{aligned}
$$

Théorème 2.1.7 ([1])

Pour tout arbre $t: D_{1}^{*} \rightarrow \Sigma_{1}$, on a $t \in L\left(\mathcal{A}_{2} \circ \mathcal{A}_{1}\right)$ si et seulement s'il existe un calcul acceptant $\rho:\left(D_{1} \times Q_{1}^{\exists}\right)^{*} \rightarrow Q_{1}^{\forall}$ de l'automate $\mathcal{A}_{1}$ sur l'arbre $t$ tel que $\rho \in L\left(\mathcal{A}_{2}\right)$. 
Remarque 2.1.8 La preuve de ce théorème, technique, qui valide la notion même de composition séquentielle, ne pose pas de difficulté particulière. Elle est donc omise ici. On peut en trouver une version détaillée dans la thèse du premier auteur [1]. Retenons cependant qu'il est crucial que l'automate $\mathcal{A}_{2}$ soit non alternant. Le théorème de simulation de Muller et Schupp (Théorème 2.1.5) nous assure cependant qu'une telle normalisation est toujours possible, quoiqu'avec un nombre d'état exponentiellement plus grand.

\subsection{Jeux à condition de gains externes}

Notre objectif est de mettre en évidence et d'exploiter les rapports étroits qui existent entre les automates d'arbres et les jeux distribués.

Cet objectif passe tout d'abord par la définition de jeux distribués dont les conditions de gain seraient exprimées de façon externe au jeu distribué à l'aide d'un automate qui spécifie directement le langage des stratégies de l'équipe des processus qui seront gagnants.

\section{Définition 2.2.1 (Condition de gain externe)}

Un jeu avec condition de gain externe est un $n$-uplet

$$
G=\left\langle P, E, T_{P}, T_{E}, e_{0}, \mathcal{A}\right\rangle
$$

où $\left\langle P, E, T_{P}, T_{E}\right\rangle$ est une arène de jeux, $e_{0} \in E$ est une position initiale dans cette arène, et $\mathcal{A}$ est un automate de $(P, E)$-arbre. Dans un tel jeu, on dira que le joueur processus admet une stratégie gagnante lorsqu'il admet une stratégie $\sigma$ dont l'arbre $t_{\sigma}\left(e_{0}\right)$ induit par les parties issues de la position $e_{0}$ et compatibles avec la stratégie $\sigma$ appartient au langage $L(\mathcal{A})$ des arbres acceptés par l'automate $\mathcal{A}$.

Cette définition s'étend sans difficulté aux arènes distribuées, définissant ainsi une notion de jeux distribués avec condition externe.

Dans la suite, afin d'éviter toute confusion, un jeu avec condition de gain définie comme dans la section 1.3 sera appelé jeu avec condition de gain interne.

Remarque 2.2.2 Ce type de condition de gain définie sur les stratégies plutôt que sur les parties elles-mêmes peut sembler curieuse. Elle apparaît cependant naturellement dans les jeux distribués où chaque arène locale agit intuitivement comme une condition supplémentaire, arborescente, sur les stratégies locales utilisables dans les autres arènes.

Nous allons maintenant démontrer que les jeux avec condition de gain externe sont, pour l'essentiel, équivalents aux jeux avec condition interne, les conditions externes pouvant être internalisées au prix de l'ajout d'un joueur Processus.

\section{Théorème 2.2.3 (Internalisation)}

Pour tout jeu distribué $G$ à $n$ processus avec condition de gain externe $\mathcal{A}$ il existe un jeu distribué $G^{\prime}$ à $n+1$-processus avec condition de gain interne avec $G^{\prime}[1, \ldots, n]=G$, tel que l'équipe des processus admet une stratégie gagnante 
$\sigma$ dans le jeux $G$ si et seulement si l'équipe des processus admet une stratégie gagnante de la forme $\sigma \otimes \sigma^{\prime}$ dans le jeu $G^{\prime}$.

En particulier, en se restreignant aux stratégies distribuées, l'équipe des processus admet une stratégie distribuée gagnante dans le jeu $G$ si et seulement si elle admet une stratégie distribuée gagnante dans le jeu $G^{\prime}$.

Preuve. La structure de l'énoncé nous permet de nous restreindre, sans perte de généralité, à un jeu $G$ à deux joueurs (et donc un seul processus). Soit donc $G=\left\langle P, E, T_{P}, T_{E}, e_{0}, \mathcal{A}\right\rangle$ un tel jeu muni d'une condition de gain externe $\mathcal{A}=$ $\left\langle Q^{\forall} \uplus Q^{\exists}, P, E, q_{0}, \delta=\delta^{\forall} \cup \delta^{\exists}, A c c\right\rangle$.

L'arène distribuée à deux processus $G^{\prime}=\left\langle P^{\prime}, E^{\prime}, T_{P}^{\prime}, T_{E}^{\prime}, e_{0}^{\prime}, \mathcal{W}^{\prime}\right\rangle$ est définie de la façon suivante.

Les positions sont définies par $P^{\prime}=\left(E \times\left(Q^{\exists} \times E\right)\right) \cup\left(P \times\left(Q^{\exists} \times P\right)\right)$ et $E^{\prime}=\left(E \times Q^{\exists}\right) \cup\left(E \times Q^{\forall}\right)$. La position initiale est définie par $e_{0}^{\prime}=\left(e_{0}, q_{0}\right)$. La condition de gain est définie par $\mathcal{W}^{\prime}=\left\{w \in\left(E^{\prime} . P^{\prime}\right)^{\omega} \mid \pi_{Q^{\forall} \cup Q^{\Xi}}(w) \in A c c\right\}$. Les coups sont définis (par séquences de quatre coups successifs) de la façon suivante :

1. d'une position de la forme $(e, q) \in E \times Q^{\exists}$ (ou bien la position initiale) : l'environnement est forcé de transmettre la valeur de $e$ au processus 2 et joue donc (de façon déterministe) dans la position de processus $(e,(q, e)) \in$ $E \times\left(Q^{\exists} \times E\right)$,

2. d'une position de la forme $(e,(q, e)) \in E \times\left(Q^{\exists} \times E\right)$ : le processus 1 étant inactif, le processus 2 (qui construit un calcul sur l'automate $\mathcal{A}$ ) choisit localement un état universel $q^{\prime} \in \delta^{\exists}(q, e)$ et l'autre processus reste inactif, atteignant ainsi la position d'environnement $\left(e, q^{\prime}\right) \in E \times Q^{\forall}$,

3. d'une position de la forme $\left(e, q^{\prime}\right) \in E \times Q^{\forall}$ : l'environnement choisit (sur $G$ ) une position $p \in T_{E}(e)$ et (dans la simulation de $\mathcal{A}$ ) un état existentiel $q_{1} \in$ $\delta^{\forall}\left(q^{\prime}, p\right)$, atteignant ainsi la position de processus $\left(p,\left(q_{1}, p\right)\right) \in P \times Q^{\exists} ;$ là encore, l'environnement est forcé de transmettre la valeur de $p$ au processus 2 ,

4. d'une position de la forme $\left(p,\left(q_{1}, p\right)\right) \in P \times\left(Q^{\exists} \times P\right)$ : le processus 1 jouant sur le jeu initial $G$, choisit une position d'environnement $e_{1} \in T_{P}(p)$, le processus 2 (codant l'automate $\mathcal{A}$ ) reste quasiment inactif en ne faisant qu'effacer la position $p$, atteignant ainsi la position d'environnement $\left(e_{1}, q_{1}\right) \in E \times Q^{\exists}$.

On vérifie par récurrence sur la longueur des parties, que cette définition couvre bien l'ensemble des positions accessibles à partir de la position initiale $e_{0}^{\prime}$. On vérifie aussi que le jeu $G^{\prime}$, restreint aux positions accessibles à partir de la position initiale, est bien un jeu distribué construit sur l'arène locale $G[1]=G$ et une arène locale $G[2]$ qui se plonge dans $G_{2}=\left\langle P_{2}, E_{2}, T_{P, 2}, T_{E, 2}\right\rangle$ définie par $E_{2}=Q^{\exists} \cup Q^{\forall}, P_{2}=Q^{\exists} \times(E \cup P), T_{E, 2}=E_{2} \times P_{2}$ (ces coups sont restreints dans la définition de $\left.T_{E}^{\prime}\right)$ et $T_{P, 2}$ défini comme l'ensemble des coups de la forme $\left((q, e), q^{\prime}\right) \in\left(Q^{\exists} \times E\right) \times Q^{\forall}$ avec $q^{\prime} \in \delta^{\exists}(q, e)$ et des coups de la forme $((q, p), q) \in\left(Q^{\exists} \times P\right) \times Q^{\exists}$ avec $q \in Q^{\exists}$ et $p \in P$. 
Supposons alors que les processus ont une stratégie $\sigma$ gagnante dans $G$. Soit $\rho:\left(Q^{\exists} \times P\right)^{*} \rightarrow Q^{\forall}$ un calcul acceptant de $\mathcal{A}$ sur $t_{\sigma}$. La stratégie locale recherché $\sigma^{\prime}:\left(E^{\prime}[2] \cdot P^{\prime}[2]\right)^{+} \rightarrow E^{\prime}[2]$ sur $G^{\prime}[2]$ est définie de la façon suivante. Pour toute partie de la forme $w \cdot(q, p)$, avec $(q, p) \in\left(Q^{\exists} \times P\right)$, la position $(q, p)$ a un unique successeur $q$ dans $G^{\prime}[2]$, et on pose $\sigma^{\prime}(w \cdot(q, p))=q$. Pour toute partie de la forme $w \cdot(q, e)$, avec $(q, e) \in\left(Q^{\exists} \times E\right)$, on pose $\sigma^{\prime}(w \cdot(q, e))=\rho\left(\pi_{Q^{\exists} \times P}(w)\right)$.

La stratégie $\sigma \otimes \sigma^{\prime}$ est non bloquante. Pour prouver cela, if suffit de montrer, par récurrence sur la longueur des parties jouées, que pour toute partie $w$ dans $G^{\prime}$ consistante avec $\sigma \otimes \sigma^{\prime}$ on a bien $\pi_{Q^{\exists} \times P}(w[2]) \in \operatorname{dom}(\rho)$. Initialement, lorsque $w=\left(q_{0}, e_{0}\right)$, on a $\pi_{Q^{\exists} \times P}(w[2])=\epsilon \in \operatorname{dom}(\rho)$. Par la suite, lorsque la partie $w$ atteint la position last $(w)=(e,(q, e)) \in E \times\left(Q^{\exists} \times E\right)$, on a, par hypothèse de récurrence, $\pi_{Q^{\exists} \times P}(w[2]) \in \operatorname{dom}(\rho)$ et donc $\sigma \otimes \sigma^{\prime}(w)=\left(e, q_{1}\right)$ avec $q_{1}=\rho\left(\pi_{Q^{\Xi} \times P}(w[2])\right.$ qui est bien définie. A partir de là, pour tout successeur $\left(p,\left(q_{2}, p\right)\right) \in P \times\left(Q^{\exists} \times P\right)$, à savoir pour toute direction $p$, pour tout état $q_{2} \in \delta^{\forall}\left(q_{1}, p\right)$, on sait que $e_{2}=t_{\sigma}\left(\pi_{P}(w) \cdot p\right)$ est bien défini car $t_{\sigma}$ est un arbre de stratégie, et que $w[1]$ est consistante avec $\sigma$. Autrement dit, $\rho\left(w[2] \cdot\left(q_{2}, p\right)\right)$ est défini. L'unique successeur à considérer pour $\left(p,\left(q_{2}, p\right)\right.$ est donc $\left(e_{2}, q_{2}\right)$, qui a pour unique successeur $\left(e_{2},\left(q_{2}, e_{2}\right)\right)$. Cela conclut l'étape de récurrence.

La stratégie $\sigma \otimes \sigma^{\prime}$ est en outre gagnante. En effet, pour toute partie consistante avec $\sigma \otimes \sigma^{\prime}$, il apparaît que les états universels portés par les positions de la partie sont bien ceux qui étiquètent les noeuds successifs de la branche correspondante dans le calcul $\rho$. Par conséquent, dans le cas d'une partie infinie $w$ consistante avec $\sigma \otimes \sigma^{\prime}$, on a $\theta\left(\pi_{Q}(w[2])\right) \in A c c$ (car $\rho$ est acceptant), et donc $w \in \mathcal{W}^{\prime}$.

Réciproquement, supposons que les processus ont une stratégie gagnante $\sigma \otimes$ $\sigma^{\prime}$ dans $G^{\prime}$ : il s'agit maintenant d'exhiber un calcul acceptant $\rho:\left(Q^{\exists} \times P\right)^{*} \rightarrow$ $Q^{\forall}$ de $\mathcal{A}$ sur $t_{\sigma}$, afin de démontrer que $\sigma$ est une stratégie gagnante dans $G$.

On peut remarquer tout d'abord que $\sigma$ est non bloquante : en effet, les restrictions imposées aux coups de l'environnement dans l'arène de $G^{\prime}$ ne contraignent pas les coups possibles pour l'environnement sur $G^{\prime}[1]$.

Soient $t_{\sigma}: P^{*} \rightarrow E$ et $t_{\sigma^{\prime}}:\left(Q^{\exists} \times(P \cup E)\right)^{*} \rightarrow Q^{\exists} \cup Q^{\forall}$ les arbres des stratégies $\sigma$ et $\sigma^{\prime}$. On définit la fonction $g:\left(Q^{\exists} \times P\right)^{*} \rightarrow P^{\prime *}$ par récurrence en posant $g(\epsilon)=\left(e_{0},\left(q_{0}, e_{0}\right)\right)$ et $g(w \cdot(p, q))=g(w) \cdot(p,(q, p)) \cdot(e,(q, e))$ où $e=t_{\sigma}\left(\pi_{P}(w) \cdot p\right)$.

En posant $\rho=\pi_{2} \circ t_{\sigma \otimes \sigma^{\prime}} \circ g$ (ou bien, de façon équivalente, $\rho=t_{\sigma^{\prime}} \circ \pi_{2} \circ g$, car il n'y a pas de coups asynchrones pour le processus $n+1$ dans $G$ ), on obtient bien un calcul acceptant de $\mathcal{A}$ sur $\sigma$.

Remarque 2.2.4 Lorsque le jeu $G$ est un jeu simple (à deux joueurs) avec condition de gain externe, cette internalisation de la condition de gain peut être réécrite de telle sorte que le jeu $G^{\prime}$ résultant est un jeu simple (à deux joueurs) qui peut donc être résolu. Remarquons au passage que lorsque Acc est une condition $\omega$-régulière, la condition de gain $\mathcal{W}$ construite pour $G^{\prime}$ est bien aussi $\omega$-régulière. 


\subsection{Théorème d'externalisation}

Dans le jeu produit à partir de l'architecture pipeline évoquée dans la première partie, on constate que, à chaque instant, lorsque toutes les stratégies locales des processus sont connues, le processus d'indice $i$ est capable de prédire la position courante sur toutes les arènes d'indice supérieur. En effet il connaît l'entrée fournie à la sous-architecture composée de ces processus puisqu'il la fournit lui-même.

Cette observation suggère que, dès lors qu'un processus dans un jeu distribué admet, explicitement ou par inférence, une certaine connaissance du reste du jeu, il peut se comporter comme un automate d'arbres qui peut reconstruire le comportement global du système.

Autrement dit, sous certaines conditions de connaissance par un processus du reste du jeu distribué, il devrait exister une construction inverse à l'internalisation d'une condition de gain externe nous permettant, tout au contraire, d'externaliser voire de combiner avec une condition externe déjà existante, le jeu local associé à ce processus. Ce faisant, le nombre d'arènes locales mises en oeuvre pourrait être réduit et, nous pourrions inductivement résoudre le jeu distribué. C'est ce que ne nous proposons de démontrer dans cette partie.

La notion de leader définie ci-dessous formalise la notion intuitive de connaissance par un processus de l'ensemble du jeu. Définie par projection sur les jeux distribués à deux processus, elle se généralise sans peine à un jeu distribué à $n+1$ processus.

\section{Définition 2.3.1 (Leader)}

Etant donné un jeu distribué à deux processus $G=\left\langle P, E, T_{P}, T_{E}, e_{0}, \mathcal{A}\right\rangle$, le processus 2 est appelé processus leaderălorsque, pour toute position (accessible) $e \in E$ de l'environnement, toute position $x$ et $y \in P$ des processus immédiatement accessibles à partir de $e$, i.e. avec $(e, x) \in T_{E}$ et $(e, y) \in T_{E}$,

- si $x[2]=y[2]$ alors $x[1]=y[1]$,

- si $x[2] \in E[2]$ ou $y[2] \in E[2]$ alors $x=y$.

Remarque 2.3.2 Intuitivement, le processus 2 est un leader lorsque, dès lors qu'il connaît une position d'environnement globale $e \in E$, quelque soit le coup joué par l'environnement à partir de cette position (ou bien après quelques coups jusqu'à son activation si le processus 2 est inactif dans la position $e$ ) le processus 2 peut prédire, à la lecture de sa seule position locale, la position globale atteinte.

Cette propriété locale, qui porte sur chaque coup de l'environnement, s'étend à une notion de connaissance globale de la façon suivante. Dans un jeu distribué $G=\left\langle P, E, T_{P}, T_{E}, e_{0}\right\rangle$ à deux processus dans lequel le processus 2 est leader, pour toute stratégie $\sigma$, la restriction de view 2 aux parties finies issues de $e_{0}$, terminant dans une position où le processus 2 est actif, est consistante avec la stratégie $\sigma$ est injective.

Cette observation nous conduit au résultat suivant : 
Lemme 2.3.3 Pour tout jeu distribué à deux processus $G=\left\langle P, E, T_{P}, T_{E}, e_{0}, \mathcal{W}\right\rangle$ dans lequel le processus 2 est leader, il existe un automate $\mathcal{A}_{2}$ de $(P[1], E[1])$ arbres tel que, pour toutes stratégies du ou des processus $\sigma$ sur $G$ et $\sigma_{1}$ sur $G[1]$, les propriétés suivantes sont équivalentes :

(1) il existe une stratégie $\sigma_{2} \operatorname{sur} G[2]$ telle que $\sigma=\sigma_{1} \otimes \sigma_{2}$

(2) il existe un calcul acceptant $\rho$ de l'automate $\mathcal{A}_{2}$ sur l'arbre $t_{\sigma_{1}}$ avec, de surcroît, $\rho=t_{\sigma_{2}}$.

Preuve. Nous donnons tout d'abord une construction de l'automate $\mathcal{A}_{2}$ dans le cas où les deux processus $\mathcal{A}_{1}$ et $\mathcal{A}_{2}$ restent actifs.

L'automate $\mathcal{A}_{2}=\left\langle Q_{2}, P[1], E[1], q_{0,2}, \delta_{2}, A c c_{2}\right\rangle$ est défini de la façon suivante :

$-Q_{2}^{\forall}=E ; Q_{2}^{\exists}=P[2] \cup\left\{q_{0,2}\right\}$,

$-\delta_{2}^{\forall}\left(q, p_{1}\right)=\left\{p_{2} \in Q_{2}^{\exists}:\left(q,\left(p_{1}, p_{2}\right)\right) \in T_{E}\right\} \quad\left(q \in Q_{2}^{\forall}, p_{1} \in P[1]\right)$,

$-\delta_{2}^{\exists}\left(p_{2}, e_{1}\right)=\left\{q \in Q_{2}^{\forall}: q[1]=e_{1} \wedge\left(p_{2}, q[2]\right) \in T_{P}[2]\right\} \quad\left(p_{2} \in Q_{2}^{\exists}, e_{1} \in E[1]\right)$ avec $\delta_{2}^{\exists}\left(q_{0,2}, e_{1}\right)=\left\{e_{0}[2]\right\}$,

$-A c c_{2}=Q_{2}^{\omega}$.

La correspondance énoncée ci-dessus entre les calculs de l'automate $\mathcal{A}_{2}$ sur les arbres de stratégie du processus 1 dans le jeu local $G[1]$ et les stratégies de l'équipe des processus dans le jeu $G$ est une conséquence simple de notre définition et du fait que le processus 2 est un leader dans $G$.

La preuve ne présente aucune difficulté puisqu'il ne s'agit que de vérifier que pour toute stratégie du processus 1 sur la composante $G[1]$, les calculs acceptants $\rho$ de $\mathcal{A}_{2}$ sur $t_{\sigma_{1}}$ sont tout simplement les arbres des stratégies $\sigma_{2}$ du processus 2 sur la composante $G[2]$ telle que la stratégie distribuée résultante $\sigma_{1} \otimes \sigma_{2}$ soit gagnante dans $G$.

Le cas où les processus peuvent être inactivés peut aussi être traité par réduction à un jeu synchrone comme indiqué dans la section 3.2.

Puisque le résultat précédent est vrai pour toute arène, même dans le cas ou $G[1]$ est une arène distribuée, il vient :

\section{Théorème 2.3.4 (Externalisation)}

Pour tout jeu distribué à $n+1$ processus de la forme $G=\left\langle P, E, T_{P}, T_{E}, e_{0}, \mathcal{A}\right\rangle$ avec condition de gain externe $\mathcal{A}$ et tel que le processus $n+1$ est leader dans $G$ il existe un automate de $(P[1 \cdots n], E[1 \cdots n])$-arbre $\mathcal{A}_{n+1}$ tel que les propositions suivantes sont équivalentes :

(1) les processus ont une stratégie distribuée gagnante dans le jeu $G$,

(2) les processus ont une stratégie distribuée gagnante dans le jeu $\left\langle G[1 \ldots n], e_{0}[1 \ldots n], \mathcal{A} \circ\right.$ $\left.\mathcal{A}_{n+1}\right\rangle$.

\subsection{Application aux jeux linéaires}

On s'intéresse ici au cas des architecture linéaires dans lesquelles, inductivement, chaque processus d'indice $i$ est leader sur le jeu distribué $G[1, i]$ constitué 
des arènes d'indice inférieur ou égal à $i$. Appliqué de proche en proche, le théorème d'externalisation présenté ci-dessus nous permet de réduire un jeu linéaire à un jeu à deux joueurs, démontrant ainsi que les jeux linéaires sont déterminés et que l'existence de stratégie distribuée gagnante pour les processus est décidable.

\section{Définition 2.4.1}

Etant donné un jeu distribué à $n$ processus de la forme $G=\left\langle P, E, T_{P}, T_{E}, e_{0}, \mathcal{W}\right\rangle$ on dit que le jeu $G$ est un jeu distribué linéaire lorsque, pour tout indice $i \in[1, n]$, pour toute position de l'environnement e et $f$, pour toutes positions des processus $p$ and $q \in P$ telles que $(e, p) \in T_{E}$ et $(f, q) \in T_{E}$ :

$$
\begin{aligned}
& \text { Si e }[1, i]=f[1, i] \text { et } p[i]=q[i] \in P[i] \text { ou } p[i] \in E[i] \text { ou } q[i] \in E[i] \\
& \text { alors } p[1, i]=q[1, i] \text {. }
\end{aligned}
$$

A nouveau, cette propriété (locale) de linéarité assure que, avant tout coup de l'environnement, si le processus $i$ connaît non seulement sa propre position $e[i]$ mais aussi les positions $e[1, i-1]$ de tous les processus d'indice plus petit, alors le processus $i$ connait toujours les $p[1, i-1]$ après le coup de l'environnement.

De plus, puisque les processus peuvent connaître à l'avance les stratégies jouées par chacun d'eux, cette propriété locale garantit aussi que, globalement, à partir de la position initiale $e_{0}$ connue de tous les processus, étant fixé une stratégie distribuée $\sigma=\sigma_{1} \otimes \cdots \otimes \sigma_{n}$, à tout instant de toute partie compatible avec la stratégie distribuée $\sigma$ chaque processus actif connaît les positions de tous les processus d'indice inférieur.

Il vient :

\section{Théorème 2.4.2}

Les jeux distribués linéaires sont décidables. De plus, si l'équipe des processus admet une stratégie distribuée gagnante alors elle admet une stratégie distribuée gagnante finie.

Preuve. Soit $n>0$ le nombre de processus, et soit $G=\left\langle P, E, T_{E}, T_{P}, e_{0}, \mathcal{A}\right\rangle$ un jeu distribué linéaire à $n+1$ processus avec une condition de gain externe $\mathcal{A}$ présentée sous la forme d'un automate fini non alternant. Le processus $n+1$ étant leader, par application du Théorème 2.3.4, on fabrique un jeu équivalent à $n$ processus, avec condition de gain externe de la forme $\mathcal{A} \circ \mathcal{A}_{n}$ dans lequel le processus $n$ est maintenant leader. On peut donc, après application du théorème de simulation sur l'automate $\mathcal{A} \circ \mathcal{A}_{n}$ pour obtenir un automate non alternant, réitérer le procédé jusqu'à obtenir un jeu à un processus avec condition de gain externe.

Ce dernier jeu peut alors être résolu par des techniques classiques. On peut par exemple se ramener à un jeu à deux joueurs est conclure en appliquant le Théorème 1.3.4.

Remarque 2.4.3 A chaque itération, l'application du théorème de simulation à l'automate $\mathcal{A} \circ \mathcal{A}_{n}$ produit une condition externe de taille exponentiellement 
plus grande. La complexité de la résolution d'un jeu distribué linéaire est donc très grande. Ce n'est pas surprenant puisqu'on sait que ce problème est non élémentaire [23].

On vérifie facilement que les jeux induits par les architectures pipeline décrites dans la section 1.4 sont linéaires. On en conclut que le problème de la synthèse distribuée sur les architectures pipelines est décidable : on peut effectivement vérifier s'il existe des fonctions séquentielles finies (une par composant) qui réalisent, ensemble, un comportement global adéquat.

Notre approche généralise l'approche [23] en ce que les spécifications de comportements globales traitées ici peuvent être arborescentes. Notre approche généralise l'approche [11] en ce qu'on traite aussi le cas de comportements (partiellement) asynchrones. Les jeux linéaires permettent aisément de coder le cas où les processus ne produisent pas forcément de sortie à chaque entrée.

\section{Synchronisation des jeux distribués}

Dans notre définition des jeux distribués, la possibilité est parfois offerte à l'environnement d'inactiver un processus, désynchronisant ainsi l'ensemble des processus. La lecture attentive de la définition des stratégies distribuées montre par ailleurs que les processus ne s'aperçoivent même pas, si l'on peut dire, de cette désynchronisation.

Les jeux résultants, qu'on pourrait qualifier d'asynchrones, sont bien adaptés à la modélisation. Par exemple, on peut vouloir modéliser la situation où deux processus communiquent au travers d'un canal, et disposent chacun d'une mémoire interne pour stocker des résultats de calcul. Il peut s'avérer pratique de mettre un processus en attente le temps qu'une communication s'effectue. Dans d'autres cas, on peut désirer modéliser des systèmes vraiment asynchrones. Dans ce cas, les processus ne doivent pas disposer d'une horloge globale; la possibilité pour l'environnement de laisser un processus inactif permet de masquer le tic d'horloge implicite fourni par les coups successifs de l'environnement.

La présence de cet asynchronisme augmente-t-il pour autant le pouvoir d'expression des jeux distribués? Dans cette partie, nous répondons par la négative à cette question.

Plus précisément, nous démontrons que, dès lors qu'on se restreint à des stratégies à mémoire finie, tout jeu asynchrone peut être réduit en un jeu synchrone (de même taille) qui admet, essentiellement, les mêmes stratégies distribuées gagnantes. Ce résultat constitue l'essentiel d'une communication faite en 2008 [4]. Il sont par ailleurs présentés en détail dans la thèse du premier auteur [1].

\subsection{Jeux synchrones et synchronisation de jeux asynchrones}

Un jeu distribué est appelé synchrone lorsque l'environnement n'a pas la possibilité d'inactiver un processus. Formellement :

Définition 3.1.1

Un jeu distribué de $n$ processus $G=\left\langle P, E, T_{E}, T_{P}, e_{0}, \mathcal{W}\right\rangle$ est dit synchrone 
lorsque $T_{E} \subseteq E \times \prod_{i \in[1, n]} P[i]$. Dans le cas contraire, il est dit asynchrone.

Remarque 3.1.2 Dans le cas d'un jeu synchrone, l'ensemble des positions de processus accessibles depuis la position initiale est de la forme $\prod_{i \in[1, n]} P[i]$. Plus encore, pour chaque partie globale $w \in(E . P)^{*} \cdot E^{?}$, on a view $(w)=w[i]$, i.e. la vue locale d'une partie globale n'est que sa projection locale. En effet, dans chaque position de processus, tous les processus sont actifs. Dès lors, étant données $n$ stratégies locales $\left\{\sigma_{i}\right\}_{i \in[1, n]}$, la stratégie globale induite $\sigma_{1} \otimes \cdots \otimes \sigma_{n}$ est définie pour toute partie globale $w$ terminant dans une position des processus par :

$$
\sigma_{1} \otimes \cdots \otimes \sigma_{n}(w)=\left(\sigma_{1}(w[1]), \ldots, \sigma_{n}(w[n])\right)
$$

On le voit, dans le cas des jeux synchrones, les définitions se simplifient.

Dans le cas d'un jeux asynchrone ce n'est pas vrai. La vue locale d'un processus désactivé ne change pas alors même que la partie globale progresse. On modélise ainsi le fait que le processus inactivé n'a pas même conscience de son inactivation.

On va maintenant s'attacher à montrer que tout jeu distribué peut se réduire à un jeu synchrone. On pourra ainsi bénéficier à la fois de la souplesse de modélisation fournie par les jeux asynchrones ainsi que de la facilité de manipulation des jeux synchrones.

\section{Théorème 3.1.3}

Pour tout jeu $G$, on peut construire un jeu synchrone $\widetilde{G}$ de même taille que $G$ tel que les processus ont une stratégie distribuée à mémoire finie gagnante dans $G$ si et seulement si ils en ont une dans $\widetilde{G}$.

Le reste de cette section est consacré à la preuve de ce résultat.

Prenons un jeu distribué à $n$ Processus $G=\left\langle P, E, T, e_{0}, \mathcal{W}\right\rangle$. Intuitivement, la construction de $\widetilde{G}$ à partir de $G$ consiste a remplacer les états d'inactivation par des boucles d'attente active. Cependant, en faisant cela, on donne de l'information supplémentaire au joueur précédemment inactivé : bien qu'il ne change pas d'état, il peut maintenant compter ses propres inactivations. Pour compenser cela, on permet aussi à l'environnement de placer à tout instant tous les processus en état d'attente active. Un argument de saturation de la mémoire permet alors de prouver que, ainsi, les processus ne peuvent exploiter cette information supplémentaire.

Tout d'abord, pour chaque ensemble $E_{i}$, on se munit d'un ensemble équipotent $\widehat{E_{i}}$ tel que $E_{i} \cap \widehat{E_{i}}=\emptyset$; pour tout élément $e$ de $E_{i}$, on note $\widehat{e}$ son image dans $\widehat{E_{i}}$. Soit $\widehat{E}=\prod_{i \in\{1, \ldots, n\}} \widehat{E_{i}}$.

On considère le jeu synchrone $\widetilde{G}=\left\langle\widetilde{P}, \widetilde{E}, \widetilde{T}, e_{0}, \widetilde{\mathcal{W}}\right\rangle$ dont les positions sont :

$$
\widetilde{P}_{i}=P_{i} \cup \widehat{E_{i}} \quad ; \quad \widetilde{E_{i}}=E_{i} \quad(\text { pour tout } i \in\{1, \ldots n\})
$$


Pour toute position $x$ dans $P \cup E$, on note $\widehat{x}$ la position de $\widetilde{P}$ obtenue en remplaçant dans $e$ tous les composants appartenant à $E_{i}$ par leur image dans $\widehat{E_{i}}$. Précisément, cela donne :

$$
\widehat{x}[i]= \begin{cases}\widehat{x[i]} & \text { si } x[i] \in E_{i} \\ x[i] & \text { si } x[i] \in P_{i}\end{cases}
$$

La fonction qui assigne à tout élément $x$ de $P \cup E$ son image $\widehat{x}$ dans $\widetilde{P}$ est trivialement une bijection. Les coups de $\widetilde{G}$ sont définis comme suit :

$$
\begin{aligned}
\widetilde{T_{i}^{P}}= & T_{i}^{P} \cup\left\{(\widehat{e}, e) \mid e \in E_{i}\right\} \\
\widetilde{T^{E}}= & \left\{(e, \widehat{p}) \in \widetilde{E} \times \widetilde{P} \mid(e, p) \in T^{E}\right\} \\
& \cup\{(e, \widehat{e}) \mid e \in E\}
\end{aligned}
$$

On définit inductivement une fonction d'effacement des coups asynchrones cancel $:(\widetilde{E} \cdot \widetilde{P})^{*} \rightarrow(E \cdot P)^{*} \operatorname{comme~suit~:~cancel~}(\epsilon)=\epsilon, \operatorname{cancel}(w \cdot e \cdot \widetilde{p})=\operatorname{cancel}(w)$. $e \cdot p$, et cancel $(w \cdot e \cdot \widehat{e})=\operatorname{cancel}(w)((\operatorname{avec} p \in P, e \in E)$. On étend cette fonction aux mots infinis en écrivant : cancel $\left(x_{0} \cdot x_{1} \cdots\right)=\lim _{i \rightarrow \infty} \operatorname{cancel}\left(x_{0} \cdots x_{i}\right)$ (il s'agit bien d'une suite convergente au sens de la topologie préfixe sur les mots).

La condition de gain de $\widetilde{G}$ est alors définie ainsi :

$$
\widetilde{\mathcal{W}}=\text { cancel }^{-1}(\mathcal{W}) \cup(\widetilde{E} \cdot \widetilde{P})^{*} \cdot(E \cdot \widehat{E})^{\omega}
$$

La deuxième partie de cette condition ne fait que permettre à l'environnement de boucler sur des coups totalement asynchrones sans pour autant faire perdre les processus.

Remarque 3.1.4 Le graphe de l'arène de $G$ se plonge dans celui de l'arène de $\widetilde{G}$. Le graphe de l'arène de $\widetilde{G}$ n'est autre que le sous-graphe induit par ce plongement, auquel on ajoute une boucle sur chaque position de l'environnement, correspondant à un coup totalement asynchrone. D'autre part, la condition de gain de $\widetilde{G}$ n'est pas beaucoup plus compliquée que celle de $G$ : parmi les conditions de gain usuelles (cf. section 1.2), seule la condition d'accessibilité n'est pas conservée par cette construction.

Commençons par montrer qu'on peut "recopier" toute stratégie distribuée gagnante dans $G$ sur $\widetilde{G}$; il suffit pour cela de s'assurer que les stratégies locales ne tiennent pas compte des coups asynchrones qui sont joués sur leur arène.

Lemme 3.1.5 Pour toute stratégie distribuée gagnante $\sigma$ sur $G$, il existe une stratégie distribuée gagnante $\widetilde{\sigma}$ sur $\widetilde{G}$.

Preuve. Tout d'abord, pour tout $i \in\{1, \ldots, n\}$, on définit une fonction d'effacement des coups localement asynchrones cancel ${ }_{i}:\left(\widetilde{E}_{i} \cdot \widetilde{P}_{i}\right)^{*} \rightarrow\left(E_{i} \cdot P_{i}\right)^{*}$ de la façon suivante : $\operatorname{cancel}_{i}(\epsilon)=\epsilon$, et pour tout mot $w \in\left(E_{i} \cdot P_{i}\right)^{*}$, pour toutes positions $e \in E_{i}, p \in P_{i}, \operatorname{cancel}_{i}(w \cdot e \cdot p)=\operatorname{cancel}_{i}(w) \cdot e \cdot p, \operatorname{et}_{\operatorname{cancel}_{i}}(w \cdot e \cdot \widehat{e})=\operatorname{cancel}_{i}(w)$. 
Considérons une stratégie distribuée gagnante $\sigma=\sigma_{1} \otimes \cdots \otimes \sigma_{n}$ sur $G$. On définit la stratégie distribuée $\widetilde{\sigma}=\widetilde{\sigma_{1}} \otimes \cdots \otimes \widetilde{\sigma_{n}}$ définie pour tout $i \in\{1, \ldots, n\}$, pour toute partie locale $w \in \widetilde{E_{i}} \cdot\left(\widetilde{P}_{i} \cdot \widetilde{E_{i}}\right)^{*}$, pour toutes positions $p \in P_{i}, e \in E_{i}$ par :

$$
\begin{aligned}
& \widetilde{\sigma}_{i}(w \cdot p)= \begin{cases}\sigma_{i}\left(\operatorname{cancel}_{i}(w \cdot p)\right) & \text { si cancel }_{i}(w \cdot p) \in \operatorname{Dom}\left(\sigma_{i}\right) \\
\text { indéfinie } & \text { sinon }\end{cases} \\
& \widetilde{\sigma}_{i}(w \cdot \widehat{e})=e
\end{aligned}
$$

Il est clair que pour tout $i \in\{1, \ldots, n\}$ le diagramme suivant commute :

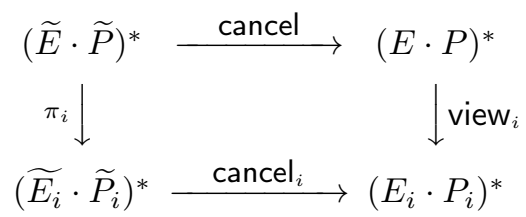

Par suite, pour toute partie globale $w \in \operatorname{Dom}(\widetilde{\sigma})$, et pour tout processus $i \in\{1, \ldots, n\}$ tel que last $(w)[i] \in P_{i}$, on a :

$$
\begin{aligned}
\widetilde{\sigma}_{i}(w[i]) & =\sigma_{i}\left(\operatorname{cancel}_{i}(w[i])\right) \\
& =\sigma_{i}\left(\operatorname{view}_{i}(\operatorname{cancel}(w))\right)
\end{aligned}
$$

Dès lors, pour toute partie infinie $w$ dans $\widetilde{G}$ consistante avec $\widetilde{\sigma}$, on a deux cas de figure : soit $w \in(E . P)^{*} .(E . \widehat{E})^{\omega}$, et est gagnante; sinon, la partie cancel $(w)$ est complète dans $G$, et consistante avec $\sigma$; elle appartient donc à $\mathcal{W}$. On a alors immédiatement $w \in \widetilde{\mathcal{W}}$. La stratégie $\widetilde{\sigma}$ est donc bien gagnante sur $\widetilde{G}$.

Il reste maintenant à prouver le lemme suivant :

Lemme 3.1.6 Pour toute stratégie distribuée à mémoire finie gagnante $\widetilde{\sigma}$ sur $\widetilde{G}$, il existe une stratégie distribuée $\sigma$ sur $G$ gagnante pour les Processus.

Concrètement, il s'agit de montrer que, même si une stratégie $\widetilde{\sigma}$ sur $\widetilde{G}$ peut localement «compter» les coups asynchrones, ce comptage est inutile. En effet, la technique de preuve va consister à saturer la mémoire de toute stratégie distribuée à mémoire finie sur $\widetilde{G}$, afin de construire une stratégie distribuée sur $G$ qui se comporte comme le ferait la stratégie sur $\widetilde{G}$ si l'environnement jouait un grand nombre de coups totalement asynchrones a chaque fois que c'est à lui de jouer.

On commence par une variation du lemme de l'étoile appliqué aux mémoires de stratégies.

Lemme 3.1.7 Pour toute arène $\langle P, E, T\rangle$, pour toute stratégie $\sigma$ à mémoire finie $\mathcal{M}=\left\langle M, m_{0}, \mu, h\right\rangle$, il existe un entier $L$ tel que pour tout élément de mémoire $m \in M$ et pour tout mot $v \in(P \cup E)^{+}$, on ait :

$$
\mu^{*}\left(m, v^{L}\right)=\mu^{*}\left(m, v^{k \cdot L}\right) \quad \text { pour tout entier } k>0
$$


Preuve. Quels que soient $m \in M$ et $v \in(P \cup E)^{+}$, soit $m_{1} \cdot m_{2} \cdots$ la suite d'états de mémoire donnée par $m_{i}=\mu^{*}\left(m, v^{i}\right)$. L'ensemble $M$ étant fini, il existe deux entiers $i$ et $j$ tels que $i<j$ et $m_{i}=m_{j}$. Soient $x_{m}^{v}=i$ et $y_{m}^{v}=j-i$. Ces deux entiers satisfont l'équation suivante :

$$
\mu^{*}\left(m, v^{x_{m}^{v}}\right)=\mu^{*}\left(m, v^{x_{m}^{v}+k \cdot y_{m}^{v}}\right) \quad \text { pour tout entier } k>0
$$

De plus, si le couple $\left(x_{m}^{v}, y_{m}^{v}\right)$ satisfait l'équation 2 , alors $\left(x_{m}^{v}+k^{\prime}, y_{m}^{v}\right)$ et $\left(x_{m}^{v}, k^{\prime} \cdot y\right)$ la satisfont aussi (quel que soit l'entier $\left.k^{\prime}\right)$. On procède comme indiqué sur le schéma ci-dessous, en itérant (a) jusqu'à avoir $x_{m}^{v}=k \cdot y_{m}^{v}$, puis en itérant (b) $k$ fois :

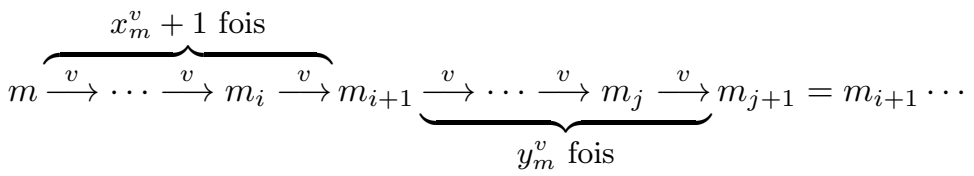

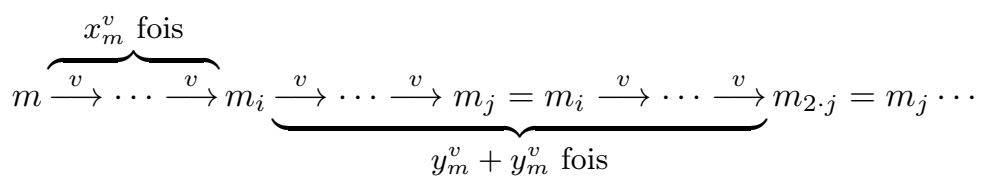

On peut par conséquent trouver un entier $L_{m}^{v}$ qui satisfait :

$$
\mu^{*}\left(m, v^{L_{m}^{v}}\right)=\mu^{*}\left(m, v^{k \cdot L_{m}^{v}}\right) \quad \text { pour tout entier } k>0
$$

Soit $\sim$ la relation binaire sur les mots de $(P \cup E)^{+}$définie par $v_{1} \sim v_{2}$ si et seulement si pour tout $m \in M, \mu^{*}\left(m, v_{1}\right)=\mu^{*}\left(m, v_{2}\right)$. Il s'agit trivialement d'une relation d'équivalence. La définition de $L_{m}^{v}$ est compatible avec $\sim$ : si $v_{1} \sim v_{2}$, alors on peut choisir de prendre $L_{m}^{v_{1}}=L_{m}^{v_{2}}$ et ainsi définir uniformément $L_{m}^{x}$ pour tout $x \in(P \cup E)^{+} / \sim$.

Remarquons à présent que l'action de chaque mot $u \in(P \cup E)^{+} \operatorname{sur}\left\langle M, m_{0}, \mu, h\right\rangle$ est caractérisée par une application $f_{u}: M \rightarrow M$, définie par $f_{u}(m)=\mu^{*}(m, u)$. En particulier, si $f_{u}=f_{v}$, alors on a $u \sim v$. Par conséquent, l'ensemble quotient $(P \cup E)^{+} / \sim$ a au plus $2^{|M|}$ éléments.

L'entier $L_{m}=\operatorname{ppcm}\left\{L_{m}^{x} \mid x \in(P \cup E)_{\sim}\right\}$ est donc bien défini. Cet entier satisfait pour tout $v \in(P \cup E)^{+}$:

$$
\mu^{*}\left(m, v^{L_{m}}\right)=\mu^{*}\left(m, v^{k \cdot L_{m}}\right) \quad \text { pour tout entier } k>0
$$

De la même façon, on définit $L=\operatorname{ppcm}\left\{L_{m} \mid m \in M\right\}$, qui satisfait l'équation 1 pour tous $m \in M$ et $v \in(P \cup E)^{+}$, ce qui conclut la preuve.

Dans le cas où l'on considère une mémoire de stratégie distribuée, le résultat précédent peut être encore affiné.

Lemme 3.1.8 Pour toute arène distribuée à $n$ Processus $\langle P, E, T\rangle$, pour toute stratégie distribuée à mémoire finie $\sigma$ avec pour mémoires $\left(\mathcal{M}_{i}=\left\langle M_{i}, m_{0, i}, \mu_{i}, h_{i}\right\rangle\right)_{i \in\{1, \ldots, n\}}$, 
il existe un entier $L$ tel que pour tout entier $i \in\{1, \ldots, n\}$, pour tout élément de mémoire $m \in M_{i}$ et pour tout mot $v \in\left(P_{i} \cup E_{i}\right)^{+}$, on ait :

$$
\mu_{i}^{*}\left(m, v^{L}\right)=\mu_{i}^{*}\left(m, v^{k \cdot L}\right) \quad \text { pour tout entier } k>0
$$

Preuve. Pour tout entier $i \in\{1, \ldots, n\}$, le lemme 3.1 .7 nous permet de dire qu'il existe un entier $L_{i}$ satisfaisant :

$$
\mu^{*}\left(m, v^{L_{i}}\right)=\mu^{*}\left(m, v^{k \cdot L_{i}}\right) \quad \text { pour tout entier } k>0
$$

L'entier $L=\operatorname{ppcm}\left\{L_{i} \mid i \in\{1, \ldots, n\}\right\}$ satisfait alors l'équation 5 .

Supposons qu'on dispose d'une stratégie distribuée à mémoire finie $\widetilde{\sigma}=$ $\widetilde{\sigma_{1}} \otimes \cdots \otimes \widetilde{\sigma_{n}}$ gagnante sur $\widetilde{G}$, avec les mémoires locales suivantes :

$$
\widetilde{\mathcal{M}_{i}}=\left\langle\widetilde{M}_{i}, \widetilde{m_{0, i}} \in \widetilde{M}_{i}, \widetilde{\mu_{i}}: \widetilde{M}_{i} \times\left(\widetilde{P}_{i} \cup \widetilde{E}_{i}\right) \rightarrow \widetilde{M}_{i}, \widetilde{h_{i}}: \widetilde{M}_{i} \times \widetilde{P}_{i} \rightarrow \widetilde{E_{i}}\right\rangle
$$

(pour $(i \in\{1, \ldots, n\})$.

On peut appliquer le résultat du lemme 3.1 .8 pour déduire la propriété suivante : il existe un entier $L$ tel que pour tout Processus $i \in\{1, \ldots, n\}$, pour tout élément de mémoire $m$ de $\widetilde{M}_{i}$, pour toute position $e \in \widetilde{E}_{i}$, on ait :

$$
\mu_{i}^{*}\left(m,(e \cdot \widehat{e})^{L}\right)=\mu_{i}^{*}\left(m,(e \cdot \widehat{e})^{k \cdot L}\right) \quad \text { pour tout } k \in \mathbb{N}
$$

On considère la stratégie distribuée à mémoire finie sur $G: \sigma=\sigma_{1} \otimes \cdots \otimes \sigma_{n}$, avec les mémoires locales $\mathcal{M}_{i}=\left\langle M_{i}, m_{0, i}, \mu_{i}, h_{i}\right\rangle$, où $M_{i}=\widetilde{M_{i}}, m_{0, i}=\widetilde{m_{0, i}}, h_{i}=$ $\widetilde{h_{i}}$, et :

$$
\begin{aligned}
& \mu_{i}(m, e)={\widetilde{\mu_{i}}}^{*}\left(m, e \cdot(\widehat{e} \cdot e)^{2 \cdot L-1}\right) \\
& \mu_{i}(m, p)=\widetilde{\mu_{i}}(m, p)
\end{aligned}
$$

On va montrer que $\sigma$ est gagnante sur $G$. Tout d'abord, considérons la fonction fill: $(E \cdot P)^{*} \rightarrow(\widetilde{E} \cdot \widetilde{P})^{*}$ définie par :

$$
\text { fill }\left(e_{0} \cdot p_{0} \cdot e_{1} \cdot p_{1} \cdots p_{n}\right)=e_{0} \cdot\left(\widehat{e_{0}} \cdot e_{0}\right)^{2 \cdot L-1} \cdot \widehat{p_{0}} \cdot e_{1} \cdot\left(\widehat{e_{1}} \cdot e_{1}\right)^{2 \cdot L-1} \cdot \widehat{p_{1}} \cdots \widehat{p_{n}}
$$

On étend fill aux mots infinis de la même façon que cancel.

Remarque 3.1.9 La fonction fill est clairement une fonction partielle des parties de $G$ and les parties de $\widetilde{G}$. Il est en outre aisé de voir que cancel o fill est la fonction identité, et donc que si fill $(w) \in \widetilde{A c c}$, alors $w \in A c c$.

Le lemme qui suit constitue le cœur de la preuve, en ce sens qu'il démontre que $\sigma$ se comporte sur $G$ comme le ferait $\widetilde{\sigma}$ sur $\widetilde{G}$ si l'environnement faisait $2 \cdot L-1$ coups totalement asynchrones chaque fois que c'est à lui de jouer.

Lemme 3.1.10 Pour toute partie infinie $w$ dans $G$ consistante avec $\sigma$, la partie fill $(w)$ est consistante avec $\widetilde{\sigma}$. 
Preuve. Soit $w=e_{0} \cdot p_{0} \cdot e_{1} \cdot p_{1} \cdots$. On note $w_{i}$ le préfixe $e_{0} \cdots e_{i}$ de $w$ (pour tout entier $i \geq 0$ )

Il suffit alors de montrer que pour tout entier $i \geq 0$, et pour chaque processus $k$ actif après $w_{i}\left(\right.$ c'est-à-dire $\left.p_{i}[k] \in P_{k}\right)$, on a $: \sigma_{k}\left(\operatorname{view}_{k}\left(w_{i}\right)\right)=\widetilde{\sigma_{k}}\left(\right.$ fill $\left.\left(w_{i}\right)[k]\right)$.

Étant donné que $\sigma$ et $\widetilde{\sigma}$ ne diffèrent que sur les fonctions de mise à jour des mémoires locale, il suffit de prouver que les mémoires locales de $\sigma$ et de $\widetilde{\sigma}$ coïncident pour tout préfixe $w_{i}$ et son image fill $\left(w_{i}\right)$ dans $\widetilde{G}$; plus précisément, on veut vérifier l'égalité suivante pour tout entier $i \geq 0$, et pour tout $k \in\{1, \ldots, n\}$ tel que $p_{i}[k] \in P_{k}$ :

$$
\mu_{k}^{*}\left(m_{0, k}, \operatorname{view}_{k}\left(w_{i}\right)\right)={\widetilde{\mu_{k}}}^{*}\left(m_{0, k}, \text { fill }\left(w_{i}\right)[k]\right)
$$

On procède par récurrence sur la longueur des préfixes $w_{i}$ de $w$. Pour la base de la récurrence, pour tout $k$ on a :

$$
\begin{array}{rlrl}
\mu_{k}^{*}\left(m_{0, k}, \operatorname{view}_{k}\left(w_{1}\right)\right) & =\mu_{k}\left(m_{0, k}, e_{0}[k]\right) & \\
& \left.={\widetilde{\mu_{k}}}^{*}\left(m_{0, k}, e_{0}[k] \cdot \widehat{e_{0}[k]} \cdot e_{0}[k]\right)^{2 \cdot L-1}\right) & & \text { définition de } \mu_{k} \\
& ={\widetilde{\mu_{k}}}^{*}\left(m_{0, k}, \text { fill }\left(e_{0}\right)[k]\right) & & \text { définition de fill }
\end{array}
$$

Par la suite, si $i$ est un entier positif, supposons que l'égalité 8 est vérifiée pour tous les préfixes $w_{j}$ de $w_{i}$, avec $0 \leq j<i$, et pour tout $k \in\{1, \ldots, n\}$ tel que $p_{j}[k] \in P_{k}$.

Pour tout $k \in\{1, \ldots, n\}$ tel que $p_{i}[k] \in P_{k}$, soit $j$ le plus grand entier strictement inférieur à $i$ tel que $p_{j}[k] \in P_{k}$ (entre $p_{j}$ et $p_{i}$, l'environnement ne joue que des coups asynchrones sur l'arène du processus $k$ ). Cette situation est résumée par le schéma suivant, en posant $p=p_{j}[k]$ et $e=e_{j+1}[k]$ :

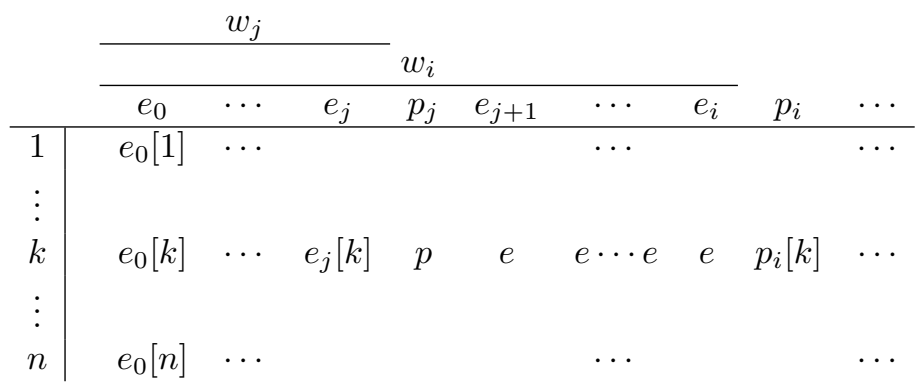

Comme on le voit, on a $w_{i}[k]=w_{j}[k] \cdot p \cdot e^{2(i-j)-1}$. En outre, on peut décomposer fill $\left(w_{i}\right)$ comme suit :

$$
\text { fill }\left(w_{i}\right)=\text { fill }\left(w_{j}\right) \cdot \widehat{p_{j}} \cdot e_{j+1} \cdot\left(\widehat{e_{j+1}} \cdot e_{j+1}\right)^{2 L-1} \cdot \widehat{p_{j+1}} \cdots e_{i} \cdot\left(\widehat{e_{i}} \cdot e_{i}\right)^{2 L-1}
$$

De plus, en utilisant le fait que $p_{\ell}[k]=e_{\ell}[k]=e_{i}[k]=e($ avec $j<\ell<i)$, on obtient la décomposition suivante pour fill $\left(w_{i}\right)[k]$ :

$$
\text { fill }\left(w_{i}\right)[k]=\operatorname{fill}\left(w_{j}\right)[k] \cdot p \cdot e \cdot(\widehat{e} \cdot e)^{2 L-1} \cdot \overbrace{\widehat{e} \cdot e \cdot(\widehat{e} \cdot e)^{2 L-1} \cdots \widehat{e} \cdot e \cdot(\widehat{e} \cdot e)^{2 L-1}}^{i-j-1 \text { fois }}
$$


On peut manipuler un peu cette expression :

$$
\begin{aligned}
\text { fill }\left(w_{i}\right)[k] & =\operatorname{fill}\left(w_{j}\right)[k] \cdot p \cdot e \cdot(\widehat{e} \cdot e)^{2 L-1} \cdot\left(\widehat{e} \cdot e \cdot(\widehat{e} \cdot e)^{2 L-1}\right)^{i-j-1} \\
& =\operatorname{fill}\left(w_{j}\right)[k] \cdot p \cdot e \cdot(\widehat{e} \cdot e)^{2 L-1} \cdot(\widehat{e} \cdot e)^{2(i-j-1) L} \\
& =\operatorname{fill}\left(w_{j}\right)[k] \cdot p \cdot e \cdot(\widehat{e} \cdot e)^{L-1} \cdot(\widehat{e} \cdot e)^{(2 i-2 j-1) L}
\end{aligned}
$$

Soit $m=\mu_{k}^{*}\left(m_{0, k}, \operatorname{view}_{k}\left(w_{j}\right)\right)$. Par hypothèse d'induction, $m={\widetilde{\mu_{k}}}^{*}\left(m_{0, k}\right.$, fill $\left.\left(w_{j}\right)[k]\right)$.

Dès lors, on peut démontrer l'hypothèse d'induction pour $w_{i}$; on posant $m^{\prime}=\mu_{k}^{*}\left(m_{0, k}, \operatorname{view}_{k}\left(w_{i}\right)\right)$, on obtient :

$$
\begin{aligned}
m^{\prime} & =\mu_{k}^{*}(m, p \cdot e) & & \\
& ={\widetilde{\mu_{k}}}^{*}\left(m, p \cdot e \cdot(\widehat{e} \cdot e)^{2 L-1}\right) & & \text { (définition de } \left.\mu_{k}\right) \\
& ={\widetilde{\mu_{k}}}^{*}\left(m, p \cdot e \cdot(\widehat{e} \cdot e)^{L-1} \cdot(\widehat{e} \cdot e)^{L}\right) & & \\
& ={\widetilde{\mu_{k}}}^{*}\left(m, p \cdot e \cdot(\widehat{e} \cdot e)^{L-1} \cdot(e \cdot \widehat{e})^{(2 i-2 j-1) L}\right) & & \text { (équation } 7) \\
& ={\widetilde{\mu_{k}}}^{*}\left(m_{0, k}, \text { fill }\left(w_{j}\right)[k] \cdot p \cdot e\right. & & \\
& \left.\cdot(\widehat{e} \cdot e)^{L-1} \cdot(e \cdot \widehat{e})^{(2 i-2 j-1) L}\right) & & \text { (hyp. d'induction) } \\
& ={\widetilde{\mu_{k}}}^{*}\left(m_{0, k}, \text { fill }\left(w_{i}\right)[k]\right) & & \text { (équation 9) }
\end{aligned}
$$

La partie infinie fill $(w)$ dans $\widetilde{G}$ est donc bien consistante avec $\widetilde{\sigma}$.

Sachant que $\widetilde{\sigma}$ est gagnante, et en vertu de la remarque 3.1.9, cela suffit pour prouver que $\sigma$ est gagnante, ce qui démontre le lemme 3.1.6.

Remarque 3.1.11 La stratégie $\sigma$ n'est pas plus complexe que $\widetilde{\sigma}$. De fait, elle utilise une mémoire de même taille.

\subsection{Synchronisation des jeux linéaires}

Comme nous l'avons déjà évoqué, le problème de la synthèse distribuée est, en général, indécidable [20,22]. L'existence d'une stratégie distribuée gagnante pour les processus dans les jeux distribués et donc tout aussi indécidable. Cette indécidabilité est même quasi immédiate puisqu'elle est déjà vraie dans le cas de jeux distribués a deux processus avec condition d'accessibilité $(\mathcal{W}=\emptyset)$ ou de sûreté $\left(\mathcal{W}=(E+P)^{\omega}\right)[9]$. Cependant, dès lors que les jeux distribués satisfont la condition de linéarité le problème de la résolution des jeux distribués devient décidable.

A la lumière de cette classe de jeux décidables, la réduction des jeux asynchrones en jeux synchrones que nous proposons ci-dessus n'est pas satisfaisante. En introduisant un non-déterminisme global de l'environnement cette réduction détruit en particulier toute propriété de linéarité : alors que le flot d'information peut être linéaire dans un jeux $G$ asynchrone il n'a aucune raison de le rester dans le jeu synchrone équivalent $\widetilde{G}$.

Nous proposons dans cette section une modification de notre construction qui permet de préserver cette linéarité.

Plus généralement, les cas de jeux décidables, comme le cas des jeux linéaires, s'appuient sur la capacité des processus d'inférer, à la volée, une connaissance 
des positions de jeux dans quelques autres arènes locales. La construction que nous proposons ici consiste, après avoir synchronisé un jeux asynchrone, à recréer par une opération de normalisation les relations de connaissance entre processus qui existaient avant l'application de la synchronisation. Présentée dans le cas des jeux linéaires, cette construction se généralise sans peine à tout schéma de connaissance implicite qui sera alors explicité. Les algorithmes de résolution des jeux qui s'appuieraient sur cette relation de connaissance pourront donc toujours être appliqués après synchronisation et normalisation.

\section{Définition 3.2.1}

Etant donné un jeu distribué à n-processus $G=\left\langle P, E, T_{P}, T_{E}, e_{0}, \mathcal{W}\right\rangle$, on définit le jeu $\operatorname{lin}(G)=\left\langle P^{\prime}, E^{\prime}, T_{P}^{\prime}, T_{E}^{\prime}, e_{0}^{\prime}, \mathcal{W}^{\prime}\right\rangle$, appelé linéarisation du jeu $G$, comme étant le jeu défini à partir du jeu $G$ de la façon suivante :

1. pour tout $i \in[1, n]$ :

(a) $P_{i}^{\prime}=P[1, i]-E[1, i]+\left\{\perp_{i}\right\}$,

(b) $E_{i}^{\prime}=E[1, i]$

(c) $T_{P, i}^{\prime}=T_{P}[1, i] \cap\left(P_{i}^{\prime} \times E_{i}^{\prime}\right)$,

2. et pour tout $e \in E^{\prime}=\prod_{i \in[1, n]} E_{i}^{\prime}$ :

(a) soit la position e est cohérente avec le jeu $G$ au sens où, pour tout $i \in[1, n]$, on a $e[i]=(e[n])[1, i]$, alors nous posons $(e, p) \in T_{E}^{\prime}$ pour toute position $p \in P^{\prime}$ telle que $\forall i \in[1, n],(e[i], p[i]) \in T_{E}[1, i]$,

(b) soit la position e est incohérente et nous posons $(e, \perp) \in T_{E}^{\prime}$ avec $\perp=\left(\perp_{1}, \cdots, \perp_{n}\right)$.

3. $e_{0}^{\prime}=\left(e_{0}[1], e_{0}[1,2], \cdots, e_{0}[1, n-1], e_{0}[1, n]\right)$,

4. et $\mathcal{W}^{\prime}=\left\{w \in\left(P^{\prime}+E^{\prime}\right)^{\omega}: w[n] \in \mathcal{W}\right\}$.

Remarque 3.2.2 Dans le jeu $\operatorname{lin}(G)$, chaque fois que les processus atteignent une position incohérente, l'environnement ne peut plus que jouer dans la position $\perp$ où les processus perdent. Il apparaît donc que les positions pertinentes du jeu, c'est-à-dire les positions où l'équipe des processus jouera, ne pourront être que des positions cohérentes, c'est-à-dire toute position $x \in E^{\prime}+P^{\prime}$ telle que, étant donné $y=x[n] \in P+E$, on a $x=(y[1], y[1,2], \cdots, y[1, n-1], y[1, n])$.

Autrement dit, dans toute position cohérente $x$ du jeu $\operatorname{lin}(G)$, pour tout indice $i \in[1, n]$, le processus $i$ connaît explicitement la position $x[j]$ de tout processus $j$ tel que $1 \leq j \leq i$. Ainsi, $\operatorname{lin}(G)$ est non seulement un jeu linéaire, mais cette linéarité est explicite.

Formellement, les stratégies distribuées dans les jeux $G$ et $\operatorname{lin}(G)$ correspondent les unes aux autres de la façon suivante.

Lemme 3.2.3 Pour toute stratégie distribuée gagnante $\sigma_{1} \otimes \cdots \otimes \sigma_{n}$ dans le jeu $G$, la stratégie distribuée $\sigma_{1}^{\prime} \otimes \cdots \otimes \sigma_{n}^{\prime}$ dans le jeu $\operatorname{lin}(G)$ définie, pour tout $i \in[1, n]$, par $\sigma_{i}^{\prime}=\sigma_{1} \otimes \cdots \otimes \sigma_{i}$, est une stratégie distribuée gagnante dans le jeu $\operatorname{lin}(G)$. 
En général, il n'y pas de construction inverse. En effet, les jeux de la forme $\operatorname{lin}(G)$ sont linéaires. L'existence de stratégie distribuée gagnante y est donc décidable ce qui n'est pas le cas en général. Cependant, lorsque le jeux $G$ est linéaire, la construction inverse est possible.

Lemme 3.2.4 Pour tout jeu distribué linéaire $G$, pour toute stratégie distribuée gagnante $\sigma_{1}^{\prime} \otimes \cdots \otimes \sigma_{n}^{\prime}$ dans le jeu $\operatorname{lin}(G)$ il existe une stratégie distribuée gagnante $\sigma_{1} \otimes \cdots \otimes \sigma_{n}$ dans le jeu $G$ telle que, pour tout $i \in[1, n]$, on ait $\sigma_{i}^{\prime}=\sigma_{1} \otimes \cdots \otimes \sigma_{i}$.

Preuve. Observons tout d'abord que, parce que la stratégie $\sigma_{1}^{\prime} \otimes \cdots \otimes \sigma_{n}^{\prime}$ est gagnante dans le jeu $\operatorname{lin}(G)$ elle ne passe que sur des positions cohérentes. Sans perte de généralité, on peut supposer que les stratégies locales suivies par les processus sont elles-même cohérentes. Plus précisément, on peut supposer que, pour tous indices $i$ et $j$ tels que $1 \leq i<j \leq n$, pour toute partie globale finie $w$ dans le jeu $\operatorname{lin}(G)$, nous avons $\sigma_{i}^{\prime}\left(\operatorname{view}_{i}(w)\right)=\sigma_{j}^{\prime}\left(\operatorname{view}_{j}(w)\right)[1, i]$

Cela étant dit, la propriété énoncée vient immédiatement de l'étude des jeux linéaires présentés précédemment (voir section ). La stratégie distribuée $\sigma_{1} \otimes \cdots \otimes \sigma_{n}$ peut être inductivement définie de la façon suivante.

Premièrement, la stratégie $\sigma_{1}$ est juste définie comme étant la stratégie $\sigma_{1}^{\prime}$. En effet, à l'exception de la position $\perp_{1}$ qui n'est de toute façon pas atteinte, les jeux locaux $\operatorname{lin}(G)[1]$ et $G[1]$ sont essentiellement les mêmes.

Ensuite, pour tout $i \in[2, n]$, la stratégie $\sigma_{i}$ est inductivement définie à partir de la stratégie $\sigma_{i-1}^{\prime}=\sigma_{1} \otimes \cdots \otimes \sigma_{i-1}$ et de la stratégie $\sigma_{i}^{\prime}$ en mimant, à partir de la connaissance initiale de la position $e_{0}$, de la connaissance de stratégie $\sigma_{i-1}^{\prime}$ et de la partie localement jouée $w_{i}$ dans le jeu $G[i]$, la partie $w$ (unique par linéarité) qui est jouée sur la projection $G[1, i]$ du jeu $G$ et qui satisfait $w_{i}=\operatorname{view}_{i}(w)$. A partir de cette observation, nous prenons $\sigma_{i}\left(w_{i}\right)=\sigma_{i}^{\prime}(w)$. La linéarité nous assure qu'une telle simulation peut toujours être effectuée.

Il vient :

Théorème 3.2.5

Pour tout jeu distribué linéaire $G$ asynchrone et à $n$ processus, le jeu lin $(\widetilde{G})$ est linéaire et synchrone, et il est de plus équivalent au jeu $G$ au sens où les processus admettent une stratégie distribuée gagnante dans le jeu $G$ si et seulement si ils admettent une stratégie distribuée gagnante dans le jeu lin $(\widetilde{G})$.

Preuve. Tout d'abord, dans le sens direct, toute stratégie distribuée gagnante $\sigma_{1} \otimes \cdots \otimes \sigma_{n}$ induit, par composition avec la fonction cancel, une stratégie distribuée gagnante dans le jeu $\widetilde{G}$ qui, à son tour, en appliquant le Lemme 3.2.3, induit à son tour une stratégie distribuée gagnante dans le jeu $\operatorname{lin}(\widetilde{G})$.

Réciproquement, en supposant qu'il existe une stratégie distribuée gagnante à mémoire finie $\widetilde{\sigma}$ dans le jeu $\operatorname{lin}(\widetilde{G})$, il apparaît que, en utilisant des arguments de saturation semblables à ceux utilisées dans le Théorème 3.1.3 (bien que la construction ne soit pas, à strictement parler, identique), on peut fabriquer une 
stratégie distribuée gagnante $\sigma^{\prime}$ dans le jeu $\operatorname{lin}(G)$. Cette stratégie peut alors être traduite, par le Lemme 3.2.4, en une stratégie $\sigma$ pour le jeu initial $G$.

\section{Conclusion}

Nous avons proposé dans cet article une étude des jeux distribués, en énonçant et en démontrant quelques uns des théorèmes fondamentaux de leur théorie.

Nous avons présenté les rapports étroits qui existent entre la théorie des automates d'arbres alternants - incluant notamment le théorème de simulation de tout automate alternant par un automate non alternant et la notion de composition d'automate qui devient possible - et les jeux distribués. Dans le cas des jeux linéaires - jeux qui apparaissent naturellement dans le codage des architectures pipelines - nous montrons comment la théorie classique des automates d'arbres permet de résoudre ces jeux.

En s'attardant sur la capacité des jeux à décrire aussi bien des comportements synchrones qu'asynchrones, nous avons pu énoncer et démontrer une intuition bien connue des concepteurs de systèmes distribués : les différences réelles entre les systèmes asynchrones et les systèmes synchrones n'apparaissent qu'en présence de mémoire non bornée; c'est par exemple le cas dans un système de communication par files d'attente de messages, lorsqu'apparaissent des files d'attente non bornées. Le résultat présenté ici de réduction des jeux distribués asynchrones aux jeux distribués synchrones formalise cette intuition en la généralisant aux spécifications de problèmes de synthèse de stratégie distribuée.

Notre approche, qui s'affranchit d'une quelconque architecture sous-jacente à tel ou tel modèle d'architecture de systèmes concurrents, est potentiellement plus universelle que telle ou telle approche plus ad hoc. Est-elle applicable aux architectures pipelines avec spécifications locales de Madhusudan et Thiagarajan [14], ou bien aux architectures asynchrones proposées depuis (voir [5, 6] pour ne citer que les plus récentes)? Rien n'est moins sûr.

Un codage naïf des pipelines architectures décidables avec spécifications locales fait apparaître des stratégies non déterministes sur lesquelles le théorème de composition (Théorème 2.1.7) échoue. Le codage des architectures asynchrones évoquées ci-dessus est peut-être possible (l'asynchronisme non borné ne génère pas forcément un nombre infini de positions dès lors que les messages non lus ne sont pas stockés), mais les spécificités des architectures traitées, qui les rendent décidables, risquent d'être noyées dans notre modèle peut-être trop abstrait.

Quand bien même la notion d'information incomplète décrite dans notre modèle semble effectivement permettre de modéliser la notion de processus distribués, la notion de leader décrite ici semble trop restreinte. Pour poursuivre la démarche abstraite que nous avons suivie ici, il manque encore à comprendre comment cette information partielle pourrait être structurée pour conduire à de nouveaux cas de jeux distribués décidables. 


\section{Références}

[1] J. Bernet. Jeux distribués. PhD thesis, LaBRI, University of Bordeaux I, November 2006.

[2] J. Bernet and D. Janin. Tree automata and discrete distributed games. In Maciej Liśkiewicz and Rüdiger Reischuk, editors, Fundamentals of Computation Theory, volume 3623 of LNCS, pages 540-551, Lübeck, August 2005. Springer.

[3] J. Bernet and D. Janin. On distributed program specification and synthesis in architectures with cycles. In 26th IFIP W.G 6.1 Int. Conf. on Formal Techniques for Networked and Distributed Systems (FORTE), volume 4229 of $L N C S$, pages 175-190. Springer, 2006.

[4] J. Bernet and D. Janin. From asynchronous to synchronous specifications for distributed program synthesis. In Int. conf. on Current Thrends in Theo. and Prac. Comp. Science (SOFSEM), volume 4910 of LNCS, pages 162-173. Springer, 2008.

[5] T. Chatain, P. Gastin, and N. Sznajder. Natural specifications yield decidability for distributed synthesis of asynchronous systems. In SOFSEM, pages 141-152, 2009.

[6] B. Genest, H. Gimbert, A. Muscholl, and I. Walukiewicz. Asynchronous games over tree architectures. CoRR, abs/1204.0077, 2012.

[7] E. Grädel, W. Thomas, and T. Wilke, editors. Automata, Logics and Infinite Games, volume 2500 of LNCS. Springer, 2002.

[8] D. Janin. A contribution to formal methods : games, logic and automata. Habilitation à diriger les recherches, LaBRI, University of Bordeaux 1, December 2005.

[9] D. Janin. On the (high) undecidability of distributed synthesis problems. In Int. conf. on Current Thrends in Theo. and Prac. Comp. Science (SOFSEM), volume 4362 of $L N C S$, pages 320-329. Springer, 2007.

[10] O. Kupferman and M. Y. Vardi. Church's problem revisited. Bulletin of Symbolic Logic, 5 :245-263, 1999.

[11] O. Kupferman and M. Y. Vardi. Synthesizing distributed systems. In In Proc. IEEE Symposium on Logic in Computer Science (LICS'01), pages 389-398, 2001.

[12] F. Lin and M. Wonham. Decentralized control and coordination of discrete event systems with partial observation. IEEE Transactions on automatic control, 33(12) :1330-1337, 1990.

[13] P. Madhusudan. Control and synthesis of open reactive systems. $\mathrm{PhD}$ thesis, University of Madras, 2001.

[14] P. Madhusudan and P.S. Thiagarajan. Distributed controller synthesis for local specifications. In 28th International Colloquium on Automata, Languages and Programming (ICALP'01), volume 2076 of LNCS, pages 396407. Springer, 2001. 
[15] R. McNaughton. Infinite games played on finite graphs. Annals of Pure and Applied Logic, 65 :149-184, 1993.

[16] S. Mohalik and I. Walukiewicz. Distributed games. In P. K. Pandya and J. Radhakrishnan, editors, In Proc. 23th Conference on Foundations of Software Technology and Theoretical Computer Science (FSTTCS'03), volume 2914 of $L N C S$, pages 338-351. Springer, 2003.

[17] David E. Muller and Paul E. Schupp. Alternating automata on infinite trees. Theoret. Comput. Sci., 54(2-3):267-276, 1987.

[18] D.E. Muller and P.E. Schupp. Simulating alternating tree automata by non-deterministic automata: New results and new proofs of the theorems of Rabin, McNaughton and Safra. Theoret. Comput. Sci., 141 :69-107, 1995.

[19] D. Perrin and J.E. Pin. Infinite Words; Automata, Semigroups, Logic and Games, volume 141 of Pure and Applied Mathematics. Elsevier, 2004.

[20] G.L. Peterson and J.H. Reif. Multiple-person alternation. In 20th Annual IEEE Symposium on Foundations of Computer Science (FOCS'ry), pages 348-363, october 1979.

[21] G.L. Peterson, J.H. Reif, and S. Azhar. Decision algorithms for multiplayer non-cooperative games of incomplete information. Computers and Mathematics with Applications, 43 :179-206, january 2002.

[22] A. Pnueli and R. Rosner. On the synthesis of a reactive module. In In Proc. 16th ACM Symposium on Principles of Programming Languages, pages 179-190, 1989.

[23] A. Pnueli and R. Rosner. Distributed reactive systems are hard to synthesize. In In Proc. 31th IEEE Symposium on Foundations of Computer Science (FOCS'90), pages 746-757, 1990.

[24] P. J. G. Ramadge and W. M. Wonham. The control of discrete event systems. Proceedings of the IEEE, 77(1) :81-98, January 1989.

[25] A. Vincent. Synthèse de contrôleurs et stratégies gagnantes dans les jeux de parité. In Hermès, editor, Modélisation des Systèmes Réactifs, pages $87-98,2001$. 